\title{
DISCONTINUOUS GALERKIN ERROR ESTIMATION FOR LINEAR SYMMETRIZABLE HYPERBOLIC SYSTEMS
}

\author{
SLIMANE ADJERID AND THOMAS WEINHART
}

\begin{abstract}
We present an a posteriori error analysis for the discontinuous Galerkin discretization error of first-order linear symmetrizable hyperbolic systems of partial differential equations with smooth solutions. We perform a local error analysis by writing the local error as a series and showing that its leading term can be expressed as a linear combination of Legendre polynomials of degree $p$ and $p+1$. We apply these asymptotic results to show that projections of the error are pointwise $\mathcal{O}\left(h^{p+2}\right)$-superconvergent. We solve relatively small local problems to compute efficient and asymptotically exact estimates of the finite element error. We present computational results for several linear hyperbolic systems in acoustics and electromagnetism.
\end{abstract}

\section{INTRODUCTION}

In this paper, we develop an a posteriori error estimation for the discontinuous Galerkin method applied to linear symmetrizable hyperbolic systems. First-order hyperbolic systems arise in many areas of continuum physics when fundamental balance laws are formulated (such as the conservation of mass, momentum, or energy) and if other small-scale, dissipative mechanisms can be neglected. Many of these systems are symmetrizable, such as Maxwell's equations of electromagnetism, the wave equation, and the two-dimensional Euler equation modeling gas dynamics. While linear symmetrizable systems can be symmetrized with little effort, symmetrization of nonlinear symmetrizable systems such as the two-dimensional Euler equations can be expensive, in which case it is advantageous to avoid symmetrization and discretize the original symmetrizable system.

The discontinuous Galerkin (DG) finite element method was first used to solve the neutron equation [25] and ordinary differential equations [24]. Cockburn and Shu [17, 18, 19] introduced the Runge-Kutta Discontinuous Galerkin (RKDG) method to solve first-order hyperbolic systems. The solution space of DG methods consists of piecewise continuous polynomial functions. As such, it can sharply capture discontinuities in the solution. They are also locally conservative, and can handle problems with complex geometries to high order. They have a simple communication pattern between elements with a common face, which is useful for parallel computation and adaptive methods, since it is easy to construct locally

Received by the editor September 10, 2009 and, inrevised form, June 17, 2010.

2010 Mathematics Subject Classification. Primary 65M60, 65N35; Secondary 35L50.

Key words and phrases. Discontinuous Galerkin method, linear hyperbolic systems, Friedrichs symmetrizable systems, a posteriori error estimation, superconvergence.

This research was partially supported by the National Science Foundation (Grant Numbers DMS 0511806, DMS 0809262). 
refined meshes with hanging nodes. Furthermore, they exhibit strong superconvergence that can be used to estimate the discretization error.

A posteriori error estimates are used to verify solutions, guide adaptive algorithms, and stop the refinement process. An ideal estimate is (i) asymptotically exact in the sense that the error estimate in some norm approaches zero under mesh refinement at the same rate as the actual error and (ii) computationally efficient by requiring a small fraction of the solution cost. Several a posteriori DG error estimates are known for hyperbolic problems [15, 16, 21, 23. Adjerid et al. [5] developed the first asymptotically correct a posteriori DG error estimates for onedimensional linear and nonlinear hyperbolic problems. Later, Adjerid and Massey [6, 7) showed how to construct accurate error estimates for multi-dimensional scalar problems on rectangular meshes. They showed that the leading term of the DG error is spanned by two $(p+1)$-degree Radau polynomials in the $x$ and $y$ directions, respectively. Krivodonova and Flaherty 22 showed that the leading term of the local discretization error on triangles having one outflow edge is spanned by a suboptimal set of orthogonal polynomials of degree $p$ and $p+1$. They computed DG error estimates by solving local problems involving numerical fluxes, thus requiring information from neighboring inflow elements. Adjerid and Baccouch 2, 44 investigated DG methods on structured and unstructured triangular meshes with several finite element spaces to discover new superconvergence properties and compute efficient and accurate error estimates. Cheng and Shu 12, 13, 14, investigated the superconvergence of discontinuous solutions for hyperbolic and convection-diffusion problems. They observed that $p$-degree DG [12] and LDG [13] solutions stay $\mathcal{O}\left(h^{p+2}\right)$ superconvergent in the $L^{2}$ norm towards a particular projection of the exact solution for linear and nonlinear problems. In [14 they presented a general superconvergence theory for one-dimensional linear hyperbolic and convection-diffusion problems where they proved that the difference between the discontinuous solution and a particular projection of the exact solution is $\mathcal{O}\left(h^{p+3 / 2}\right)$ and grows most linearly with time. Such superconvergence results explain the observed long-time accuracy of discontinuous solutions and are an essential ingredient in proving the asymptotic exactness of a posteriori DG error estimates 3 .

In [8, 9, we developed a residual-based a posteriori error estimation for the discontinuous Galerkin method for linear symmetric hyperbolic systems. In this paper, we extend this work and develop an a posteriori error estimation for linear symmetrizable hyperbolic systems. In our analysis, we symmetrize both the hyperbolic system and its DG formulation and apply the results of [8] to the resulting symmetric system. The a posteriori error estimation procedure for symmetrizable systems is different from that for symmetric systems since the range and the null space are generally skew for symmetrizable matrices, whereas they are orthogonal for symmetric matrices; thus, we have to be careful to distinguish between the null space orthogonal and the range of these matrices. The main differences from the symmetric case can be summarized in the following points: (i) pointwise superconvergence can be observed only in the projection of the error onto $\bigcap_{i=1}^{d} \mathcal{R}\left(\left(\mathbf{A}_{i}^{s_{i}}\right)^{t}\right)$, $s_{i}=+,-$, (ii) the error estimate is obtained by splitting the leading term of the discretization error applying skew projections into the range and null space of the flux matrices, (iii) the Drazin inverse is used to define the skew projection operators, and (iv) the test function spaces for the error estimation procedure are now based on the range and null spaces of the transpose of each flux matrix which leads 
to Petrov-Galerkin problems for the error estimates. Computational results suggest that our a posteriori error estimates stay good for a relatively long time.

This manuscript is organized as follows: in $\$ 2$ we state the problem and present the weak DG formulation for symmetrizable hyperbolic systems. In $\$ 3$ we present a local error analysis for first-order multi-dimensional hyperbolic systems. We discuss our a posteriori error estimation procedure in 4 . In $\$ 5$ we validate our theory by presenting computational results for several problems in two- and three-dimensions. We conclude with a few remarks in $\$ 6$.

\section{Problem formulation}

Let $d$ be the space dimension, $\mathbf{x}=\left(x_{1}, \ldots, x_{d}\right)^{t}$ the space variable defined on a domain $\Omega=(0,1)^{d} \in \mathbb{R}^{d}$, and $t$ the time variable defined on $[0, T]$.

Let $\mathbf{u}:[0, T] \times \Omega \rightarrow \mathbb{R}^{m}$ be the true solution of the linear symmetrizable hyperbolic system

$$
\frac{\partial \mathbf{u}}{\partial t}+\sum_{i=1}^{d} \mathbf{A}_{i} \frac{\partial \mathbf{u}}{\partial x_{i}}=\mathbf{g}(t, \mathbf{x}), \mathbf{x} \in \Omega, 0<t<T,
$$

with constant coefficient matrices $\mathbf{A}_{i}, 1 \leq i \leq d$, such that there is a symmetric positive definite matrix $\mathbf{S}_{0}$ for which the matrices

$$
\mathbf{S}_{i}=\mathbf{S}_{0} \mathbf{A}_{i}, \quad 1 \leq i \leq d,
$$

are symmetric. Further, assume that $\mathbf{u}$ satisfies the following initial and boundary conditions

$$
\begin{aligned}
\mathbf{u}(0, \mathbf{x}) & =\mathbf{u}_{0}(\mathbf{x}), \mathbf{x} \in \Omega \\
\left(\sum_{i=1}^{d}\left(\nu_{i} \mathbf{A}_{i}\right)^{-}\right) \mathbf{u}(t, \mathbf{x}) & =\left(\sum_{i=1}^{d}\left(\nu_{i} \mathbf{A}_{i}\right)^{-}\right) \mathbf{u}_{B}(t, \mathbf{x}), \mathbf{x} \in \partial \Omega, 0<t<T,
\end{aligned}
$$

where $\partial \Omega$ denotes the boundary of $\Omega$ and $\boldsymbol{\nu}$ denotes the unit outward normal on $\partial \Omega$.

We call a real matrix $\mathbf{M}$ symmetrizable, if and only there is a symmetric positive definite matrix $\mathbf{S}$ such that $\mathbf{S M}$ is symmetric. By [11, $\mathbf{M} \in \mathbf{R}^{m \times m}$ is symmetrizable, if and only if it is diagonalizable over the reals, i.e.,

$$
\mathbf{M}=\mathbf{P} \operatorname{diag}\left(\lambda_{1}, \ldots, \lambda_{m}\right) \mathbf{P}^{-1}, \mathbf{P} \in \mathbb{R}^{m \times m}, \lambda_{1}, \ldots, \lambda_{m} \in \mathbb{R} .
$$

We define

$$
\begin{aligned}
\mathbf{M}^{+} & =\mathbf{P} \operatorname{diag}\left(\max \left(\lambda_{1}, 0\right), \ldots, \max \left(\lambda_{m}, 0\right)\right) \mathbf{P}^{-1}, \\
\mathbf{M}^{-} & =\mathbf{P} \operatorname{diag}\left(\min \left(\lambda_{1}, 0\right), \ldots, \min \left(\lambda_{m}, 0\right)\right) \mathbf{P}^{-1}, \text { and } \\
\operatorname{sgn}(\mathbf{M}) & =\mathbf{P} \operatorname{diag}\left(\operatorname{sgn}\left(\lambda_{1}\right), \ldots, \operatorname{sgn}\left(\lambda_{1}\right)\right) \mathbf{P}^{-1},
\end{aligned}
$$

where $\operatorname{sgn}(x), x \in \mathbb{R}$ denotes the standard signum function.

Let $\mathcal{R}(\mathbf{M})$ and $\mathcal{N}(\mathbf{M})$ denote the range and null space of $\mathbf{M}$, respectively. Using basic linear algebra, we prove that these matrices satisfy the following properties summarized in a lemma. 
Lemma 2.1. Let $\mathbf{M} \in \mathbb{R}^{m \times m}$ be symmetrizable. Then $\mathbf{M}^{+}, \mathbf{M}^{-}$and $\operatorname{sgn}(\mathbf{M})$, as defined in (2.2), satisfy the following:

$$
\begin{aligned}
& \mathbf{M}=\mathbf{M}^{+}+\mathbf{M}^{-}, \\
& \mathcal{N}\left(\mathbf{M}^{+}-\mathbf{M}^{-}\right)=\mathcal{N}(\operatorname{sgn}(\mathbf{M}))=\mathcal{N}(\mathbf{M}) \subseteq \mathcal{N}\left(\mathbf{M}^{s}\right), s=+,-, \\
& \mathcal{R}\left(\mathbf{M}^{s}\right) \subseteq \mathcal{R}(\mathbf{M})=\mathcal{R}(\operatorname{sgn}(\mathbf{M})), s=+,-, \\
& \mathbf{M}^{+} \operatorname{sgn}(\mathbf{M})=\mathbf{M}^{+}, \quad \mathbf{M}^{-} \operatorname{sgn}(\mathbf{M})=-\mathbf{M}^{-} .
\end{aligned}
$$

We select $\mathbf{g}$, the initial conditions $\mathbf{u}_{0}$ and the boundary conditions $\mathbf{u}_{B}$ such that $\mathbf{u}(t, \mathbf{x})$ lies in the space $\mathfrak{C}^{p+2}=\left[C^{2}\left([0, T], C^{p+2}(\Omega)\right)\right]^{m}$ where

$$
C^{2}\left([0, T], C^{p+2}(\Omega)\right)=\left\{\mathbf{v} \mid \frac{\partial^{k} \mathbf{v}(t, \mathbf{x})}{\partial t^{k}} \in C^{p+2}(\Omega), k=0,1,2,0<t<T\right\} .
$$

Now, let us partition the domain $\Omega=(0,1)^{d}$ into a uniform mesh $\mathcal{T}_{h}$ consisting of $N^{d}$ square elements of size $h=N^{-1}$ defined as

$$
\mathcal{T}_{h}=\left\{\prod_{i=1}^{d}\left(n_{i} h, n_{i} h+h\right): 0 \leq n_{i}<N, 1 \leq i \leq d\right\} .
$$

Let $\mathcal{P}_{p}, p \geq 0$, denote the polynomials in $\mathbf{x} \in \mathbb{R}^{d}$ with coefficients in $\mathbb{R}^{m}$ of total degree at most $p+1$ and of degree at most $p$ in each space variable $x_{1}, \ldots, x_{d}$, that is

$$
\mathcal{P}_{p}=\left\{\sum_{\boldsymbol{\alpha}} \mathbf{c}_{\boldsymbol{\alpha}} \mathbf{x}^{\boldsymbol{\alpha}}: \mathbf{c}_{\boldsymbol{\alpha}} \in \mathbb{R}^{m},|\boldsymbol{\alpha}| \leq p+1, \max _{1 \leq i \leq d} \alpha_{i} \leq p\right\} .
$$

Here, we use the finite element space

$$
\mathcal{V}_{p}^{h}=\left\{\mathbf{v}(t, \mathbf{x}):\left.\mathbf{v}\right|_{\omega} \in \mathcal{P}_{p}, \omega \in \mathcal{T}_{h}, 0 \leq t \leq T\right\} .
$$

The weak formulation of (2.1a) is obtained by multiplying (2.1a) by a test function $\mathbf{v}$, integrating over an arbitrary element $\omega \in \mathcal{T}_{h}$, and applying Green's identity to write

$$
\int_{\omega} \mathbf{v}^{t}\left(\frac{\partial \mathbf{u}}{\partial t}-\mathbf{g}\right) d \mathbf{x}=\sum_{i=1}^{d} \int_{\omega} \frac{\partial \mathbf{v}^{t}}{\partial x_{i}} \mathbf{A}_{i} \mathbf{u} d \mathbf{x}-\int_{\partial \omega} \mathbf{v}^{t} \nu_{i} \mathbf{A}_{i} \mathbf{u} d \mathbf{s}, \forall \omega \in \mathcal{T}_{h}, 0<t<T,
$$

where $\partial \omega$ denotes the boundary of $\omega$ and $\boldsymbol{\nu}$ its outward unit normal.

Since $\mathcal{V}_{p}^{h}$ allows discontinuities across element boundaries $\partial \omega$ of any element $\omega \in \mathcal{T}_{h}$, we define the traces of $\mathbf{u}_{h} \in \mathcal{V}_{p}^{h}$ on $\partial \omega$ as

$$
\mathbf{u}_{h}^{+}(t, \mathbf{x})=\lim _{\epsilon \rightarrow 0^{+}} \mathbf{u}_{h}(t, \mathbf{x}-\epsilon \boldsymbol{\nu}), \quad \mathbf{u}_{h}^{-}(t, \mathbf{x})=\lim _{\epsilon \rightarrow 0^{+}} \mathbf{u}_{h}(t, \mathbf{x}+\epsilon \boldsymbol{\nu}), \quad \mathbf{x} \in \partial \omega,
$$

where $\boldsymbol{\nu}$ denotes the unit outward normal on the boundary $\partial \omega$. We will write $\mathbf{u}_{h}=\mathbf{u}_{h}^{+}$whenever there is no confusion.

Applying the Steger-Warming numerical flux, 27,

$$
\mathbf{h}\left(\mathbf{u}_{h}^{+}, \mathbf{u}_{h}^{-}, \boldsymbol{\nu}\right)=\sum_{i=1}^{d}\left(\left(\nu_{i} \mathbf{A}_{i}\right)^{+} \mathbf{u}_{h}^{+}+\left(\nu_{i} \mathbf{A}_{i}\right)^{-} \mathbf{u}_{h}^{-}\right), \mathbf{x} \in \partial \omega, \omega \in \mathcal{T}_{h},
$$


we complete the definition of the discontinuous Galerkin method which consists of finding $\mathbf{u}_{h} \in \mathcal{V}_{p}^{h}$ such that

$$
\begin{aligned}
& \int_{\omega} \mathbf{v}^{t}\left(\frac{\partial \mathbf{u}_{h}}{\partial t}-\mathbf{g}\right) d \mathbf{x} \\
&=\sum_{i=1}^{d}\left(\int_{\omega} \frac{\partial \mathbf{v}^{t}}{\partial x_{i}} \mathbf{A}_{i} \mathbf{u}_{h} d \mathbf{x}-\int_{\partial \omega} \mathbf{v}^{t}\left(\left(\nu_{i} \mathbf{A}_{i}\right)^{+} \mathbf{u}_{h}+\left(\nu_{i} \mathbf{A}_{i}\right)^{-} \mathbf{u}_{h}^{-}\right) d \mathbf{s}\right), \\
& \omega \in \mathcal{T}_{h}, \mathbf{v} \in \mathcal{P}_{p}, 0<t<T .
\end{aligned}
$$

This yields a system of ODEs which is integrated by the embedded DormandPrince method [20] with the temporal discretization error kept much smaller than the spatial error. However, for the purpose of analyzing the behavior of the spatial discretization error, we assume exact time integration.

In the remainder of this section, we define approximations of the initial and boundary conditions $\mathbf{u}_{0}$ and $\mathbf{u}_{B}$ by functions in $\mathcal{V}_{p}^{h}$ so the approximation errors for the initial and boundary conditions have a similar behavior as the discontinuous Galerkin discretization error.

For simplicity, we only consider the approximation on the element $\omega=(0, h)^{d}$.

Let $\Delta=(0,1)^{d}$ denote the reference element and let $\Gamma$ denote its boundary.

We split $\Gamma=\bigcup_{i=1}^{d} \Gamma_{i}$, where (2.10a)

$$
\Gamma_{i}=\Gamma_{i}^{-} \cup \Gamma_{i}^{+}, \quad \Gamma_{i}^{-}=\left\{\boldsymbol{\xi} \in \Delta: \xi_{i}=0\right\}, \quad \Gamma_{i}^{+}=\left\{\boldsymbol{\xi} \in \Delta: \xi_{i}=1\right\}, \quad 1 \leq i \leq d .
$$

For $\omega=(0, h)^{d}$ with boundary $\partial \omega$, there is an affine transformation $\mathbf{x}: \Delta \rightarrow \omega$, $\mathbf{x}(\boldsymbol{\xi})=h \boldsymbol{\xi}$.

We split $\partial \omega=\bigcup_{i=1}^{d} \gamma_{i}$, where

$$
\gamma_{i}=\gamma_{i}^{-} \cup \gamma_{i}^{+}, \quad \gamma_{i}^{ \pm}=\mathbf{x}\left(\Gamma_{i}^{ \pm}\right), 1 \leq i \leq d .
$$

Let $L_{p}(\xi)$ denote the Legendre polynomial of degree $p$, as defined in [1], shifted to $[0,1]$. It is well known that $L_{p}(\xi)$ is orthogonal to all polynomials of degree not exceeding $p-1$ and satisfies

$$
\int_{0}^{1} L_{p}(\xi) L_{q}(\xi) d \xi=\frac{\delta_{p q}}{2 p+1}, \quad \int_{0}^{1} L_{p}(\xi) L_{p+1}^{\prime}(\xi) d \xi=2,
$$

where $\delta_{p q}$ is the Kronecker delta, which is equal to 1 if $p=q$ and 0 otherwise.

In order to obtain an error in the initial and boundary conditions consistent with the DG discretization error we define the operators $\pi$ and $\pi_{i}^{s}, s=+,-, 1 \leq i \leq d$, respectively, to approximate the initial conditions on $\omega=(0, h)^{d}$ and the boundary conditions on $\gamma_{i}^{s} \cap \partial \Omega$.

Let $\Pi$ denote the $L^{2}$ projection on $\omega:\left[L^{2}(\omega)\right]^{m} \rightarrow \mathcal{P}_{p}$ and let $\Pi_{i}^{s}$ denote the $L^{2}$ projection on the boundary $\gamma_{i}^{s}:\left.\left[L^{2}\left(\gamma_{i}^{s}\right)\right]^{m} \rightarrow \mathcal{P}_{p}\right|_{\gamma_{i}^{s}}, s=+,-, 1 \leq i \leq d$.

Adjerid and Weinhart [9] showed that when the initial condition is approximated by the $L^{2}$ projection $\Pi \mathbf{u}_{0}$, both superconvergence and effectivity indices are polluted near $t=0$ and all results in this manuscript are recovered at $t=\mathcal{O}(1)$. Similarly, when the boundary conditions are approximated by the $L^{2}$ projection $\Pi_{i}^{s} \mathbf{u}_{B}$, the superconvergence is polluted on elements near the boundary.

In order to reduce these pollution effects, we approximate the initial and boundary conditions such that the leading error terms are similar to those of the DG 
error. Such operators are given below as $\pi$ for the initial condition and $\pi_{i}^{s}$ for the boundary conditions:

$$
\begin{gathered}
\pi \mathbf{v}(\mathbf{x})=\Pi \mathbf{v}(\mathbf{x})+\sum_{i=1}^{d} L_{p}\left(\frac{x_{i}}{h}\right) \operatorname{sgn}\left(\mathbf{A}_{i}\right) \overline{\mathbf{c}}_{i}, \quad \overline{\mathbf{c}}_{i}=\frac{\int_{\omega} \mathbf{v}(\mathbf{x}) L_{p+1}\left(\frac{x_{i}}{h}\right) d \mathbf{x}}{\int_{\omega} L_{p+1}^{2}\left(\frac{x_{i}}{h}\right) d \mathbf{x}} . \\
\pi_{i}^{s} \mathbf{v}(\mathbf{x})=\Pi_{i}^{s} \mathbf{v}+\sum_{j \in D(i)} L_{p}\left(\frac{x_{i}}{h}\right) \operatorname{sgn}\left(\mathbf{A}_{i}\right) \overline{\mathbf{c}}_{i j}^{s},
\end{gathered}
$$

where $D(i)=\{1,2, \ldots, d\} \backslash\{i\}$, and

$$
\overline{\mathbf{c}}_{i j}^{s}=\frac{\int_{\gamma_{i}^{s}} \mathbf{v}(\mathbf{x}) L_{p+1}\left(\frac{x_{j}}{h}\right) d \mathbf{s}}{\int_{\gamma_{i}^{s}} L_{p+1}^{2}\left(\frac{x_{j}}{h}\right) d \mathbf{s}}, \quad s=+,-, j \in D(i), 1 \leq i \leq d .
$$

In our analysis we will need the following lemma proved in $[8]$ and we include it here for the sake of completeness and clarity.

Lemma 2.2. Let $\omega=(0, h)^{d}, \mathbf{v}(\mathbf{x}) \in\left[C^{p+2}(\bar{\omega})\right]^{m}$ and $\boldsymbol{\xi}=h^{-1} \mathbf{x}$. If $\Pi \mathbf{v}$ is the $L^{2}$-projection onto $\mathcal{P}_{p}$ on $\omega$ and $\pi_{i}^{s} \mathbf{v}$ as defined in (2.13a). Then there exists a positive constant $C$ independent of $h$ such that

$$
\left\|\mathbf{v}(\mathbf{x})-\Pi \mathbf{v}(\mathbf{x})-h^{p+1} \sum_{j=1}^{d} L_{p+1}\left(\frac{x_{j}}{h}\right) \mathbf{c}_{j}\right\|_{\infty, \omega} \leq C h^{p+2},
$$

$$
\left\|\mathbf{v}(\mathbf{x})-\pi \mathbf{v}(\mathbf{x})-h^{p+1} \sum_{j=1}^{d}\left(L_{p+1}\left(\frac{x_{j}}{h}\right) \mathbf{c}_{j}-L_{p}\left(\frac{x_{j}}{h}\right) \operatorname{sgn}\left(\mathbf{A}_{j}\right) \mathbf{c}_{j}\right)\right\|_{\infty, \omega} \leq C h^{p+2} .
$$

Furthermore, on the boundary $\gamma_{i}^{s}$ we have

$$
\begin{gathered}
\left\|\mathbf{v}(\mathbf{x})-\pi_{i}^{s} \mathbf{v}(\mathbf{x})-h^{p+1} \sum_{j \in D(i)}\left(L_{p+1}\left(\frac{x_{j}}{h}\right) \mathbf{c}_{j}-L_{p}\left(\frac{x_{j}}{h}\right) \operatorname{sgn}\left(\mathbf{A}_{i}\right) \mathbf{c}_{j}\right)\right\|_{\infty, \gamma_{i}^{s}} \leq C h^{p+2}, \\
s=+,-, 1 \leq i \leq d,
\end{gathered}
$$

where

$$
\mathbf{c}_{j}=\frac{1}{a_{p+1}} \frac{1}{(p+1) !} \frac{\partial^{p+1} \mathbf{v}(\mathbf{0})}{\partial x_{j}^{p+1}}, 1 \leq j \leq d,
$$

and $a_{p+1}$ denotes the coefficient of $\xi^{p+1}$ in $L_{p+1}(\xi)$.

Proof. The proof is given in 8 .

\section{LOCAL ERROR ANALYSIS}

In this section we perform a local error analysis on one element $\omega=(0, h)^{d}$ by writing the local error as a Maclaurin series and showing that its leading term can be expressed as a linear combination of Legendre polynomials of degree $p$ and $p+1$. For special hyperbolic systems where the coefficient matrices are nonsingular we show that the leading term of the error is spanned by $(p+1)$-degree Radau polynomials. 
Thus, let $\mathbf{u}_{h} \in \mathcal{P}_{p}$ satisfy the local DG formulation on $\omega=(0, h)^{d}$,

$$
\begin{array}{r}
\int_{\omega} \mathbf{v}^{t}\left(\frac{\partial \mathbf{u}_{h}}{\partial t}-\mathbf{g}\right) d \mathbf{x} \\
=\sum_{j=1}^{d}\left(\int_{\omega} \frac{\partial \mathbf{v}^{t}}{\partial x_{j}} \mathbf{A}_{j} \mathbf{u}_{h} d \mathbf{x}-\int_{\gamma_{j}} \mathbf{v}^{t}\left(\left(\nu_{j} \mathbf{A}_{j}\right)^{+} \mathbf{u}_{h}+\left(\nu_{j} \mathbf{A}_{j}\right)^{-} \mathbf{u}_{h}^{-}\right) d \mathbf{s}\right), \\
\forall \mathbf{v} \in \mathcal{P}_{p}, 0<t<T,
\end{array}
$$

subject to the initial and boundary conditions

$$
\begin{gathered}
\mathbf{u}_{h}(0, \mathbf{x})=\pi \mathbf{u}_{0}(\mathbf{x}), \quad \mathbf{x} \in \omega, \\
\left(\nu_{i} \mathbf{A}_{i}\right)^{-} \mathbf{u}_{h}^{-}(t, \mathbf{x})=\left(\nu_{i} \mathbf{A}_{i}\right)^{-} \pi_{i}^{s} \mathbf{u}(t, \mathbf{x}), \\
\mathbf{x} \in \gamma_{i}^{s}, s=+,-, 1 \leq i \leq d, 0<t<T,
\end{gathered}
$$

where $\mathbf{u}=\mathbf{u}_{B}$ on the boundary of $\Omega$.

Next, we will show that there is a symmetric system equivalent to (2.1) and that the corresponding DG formulation, as developed in [8], is equivalent to (3.1). Then we apply the results of [8] to the symmetric system.

Since $\mathbf{S}_{0}$ is symmetric positive definite, it admits a Cholesky factorization

$$
\mathbf{S}_{0}=\mathbf{R}^{t} \mathbf{R}
$$

where $\mathbf{R}$ is a positive definite matrix. We define the auxiliary matrix

$$
\mathbf{B}_{i}=\mathbf{R} \mathbf{A}_{i} \mathbf{R}^{-1}, 1 \leq i \leq d
$$

The following properties, which can be shown using basic linear algebra, will be needed in our error analysis.

Lemma 3.1. Let $\mathbf{R}$ and $\mathbf{B}_{i}, 1 \leq i \leq d$, be as defined in (3.2). Then $\mathbf{B}_{i}$ is symmetric and

$\mathbf{B}_{i} \mathbf{R}=\mathbf{R} \mathbf{A}_{i}, \quad \mathbf{B}_{i}^{s} \mathbf{R}=\mathbf{R} \mathbf{A}_{i}^{s}, s=+,-, \quad \operatorname{sgn}\left(\mathbf{B}_{i}\right) \mathbf{R}=\mathbf{R} \operatorname{sgn}\left(\mathbf{A}_{i}\right), \quad s=+,-, \quad 1 \leq i \leq d$.

Furthermore, the following holds for every $\mathbf{v} \in \mathbb{R}^{m}$ :

$$
\begin{aligned}
& \mathbf{v} \in \mathcal{N}\left(\mathbf{A}_{i}\right) \text { if and only if } \mathbf{R} \mathbf{v} \in \mathcal{N}\left(\mathbf{B}_{i}\right) \\
& \mathbf{v} \in \mathcal{R}\left(\mathbf{A}_{i}\right) \text { if and only if } \mathbf{R} \mathbf{v} \in \mathcal{R}\left(\mathbf{B}_{i}\right), \\
& \mathbf{v} \in \mathcal{N}\left(\mathbf{A}_{i}^{t}\right) \text { if and only if } \mathbf{R}^{-t} \mathbf{v} \in \mathcal{N}\left(\mathbf{B}_{i}^{t}\right)=\mathcal{N}\left(\mathbf{B}_{i}\right) \text {, and } \\
& \mathbf{v} \in \mathcal{R}\left(\mathbf{A}_{i}^{t}\right) \text { if and only if } \mathbf{R}^{-t} \mathbf{v} \in \mathcal{R}\left(\mathbf{B}_{i}^{t}\right)=\mathcal{R}\left(\mathbf{B}_{i}\right) .
\end{aligned}
$$

In the remainder of this section we show how to symmetrize the hyperbolic system and its weak DG formulation.

First, we left-multiply (2.1) by $\mathbf{R}$, substitute $\mathbf{U}=\mathbf{R u}$, and use (3.3) to obtain the symmetric system

$$
\frac{\partial \mathbf{U}}{\partial t}+\sum_{i=1}^{d} \mathbf{B}_{i} \frac{\partial \mathbf{U}}{\partial x_{i}}=\mathbf{R g}(t, \mathbf{x}), \quad \mathbf{x} \in \Omega, 0<t<T
$$


subject to the initial and boundary conditions

$$
\begin{aligned}
\mathbf{U}(0, \mathbf{x}) & =\mathbf{R u}_{0}(\mathbf{x}), \quad \mathbf{x} \in \Omega, \\
\left(\sum_{i=1}^{d}\left(\nu_{i} \mathbf{B}_{i}\right)^{-}\right) \mathbf{U}^{-}(t, \mathbf{x}) & =\left(\sum_{i=1}^{d}\left(\nu_{i} \mathbf{B}_{i}\right)^{-}\right) \mathbf{R u}(t, \mathbf{x}), \quad \mathbf{x} \in \partial \Omega, 0<t<T .
\end{aligned}
$$

Substituting $\mathbf{w}=\mathbf{R}^{-t} \mathbf{v}$ and $\mathbf{U}_{h}=\mathbf{R} \mathbf{u}_{h}$ in (3.1a) and using (3.3) yields

$$
\begin{aligned}
\int_{\omega} \mathbf{w}^{t} & \left(\frac{\partial \mathbf{U}_{h}}{\partial t}-\mathbf{R g}\right) d \mathbf{x}=\sum_{j=1}^{d}\left(\int_{\omega} \frac{\partial \mathbf{w}^{t}}{\partial x_{j}} \mathbf{B}_{j} \mathbf{U}_{h} d \mathbf{x}\right. \\
& \left.-\int_{\partial \omega} \mathbf{w}^{t}\left(\left(\nu_{i} \mathbf{B}_{i}\right)^{+} \mathbf{U}_{h}^{+}+\left(\nu_{i} \mathbf{B}_{i}\right)^{-} \mathbf{U}_{h}^{-}\right) d \mathbf{s}\right), \forall \mathbf{v} \in \mathcal{P}_{p}, 0<t<T .
\end{aligned}
$$

Left-multiplying (3.1b-c) by $\mathbf{R}$, substituting $\mathbf{U}=\mathbf{R u}$, and using (3.3) yields the initial and boundary conditions

$$
\begin{gathered}
\mathbf{U}_{h}(0, \mathbf{x})=\mathbf{R} \pi \mathbf{u}_{0}(\mathbf{x}), \quad \mathbf{x} \in \omega, \\
\left(\nu_{i} \mathbf{B}_{i}\right)^{-} \mathbf{U}_{h}^{-}(t, \mathbf{x})=\left(\nu_{i} \mathbf{B}_{i}\right)^{-} \mathbf{R} \pi_{i}^{s} \mathbf{u}(t, \mathbf{x}), \\
\mathbf{x} \in \gamma_{i}^{s}, s=+,-, 1 \leq i \leq d, 0<t<T .
\end{gathered}
$$

Next, we show that the initial and boundary conditions $\mathbf{U}_{h}(0, \mathbf{x})$ and $\mathbf{U}_{h}^{-}(t, \mathbf{x})$, respectively, may be expressed as approximations of $\mathbf{U}(0, \mathbf{x})=\mathbf{R} \mathbf{u}_{0}(\mathbf{x})$ and $\mathbf{U}^{-}(t, \mathbf{x})=\mathbf{R} \mathbf{u}^{-}(t, \mathbf{x})$.

For $\mathbf{v} \in\left[L^{2}(\omega)\right]^{m}$, we define the approximation operator

$$
\tilde{\pi} \mathbf{v}(\mathbf{x})=\Pi \mathbf{v}(\mathbf{x})+\sum_{i=1}^{d} L_{p}\left(\frac{x_{i}}{h}\right) \operatorname{sgn}\left(\mathbf{B}_{i}\right) \overline{\mathbf{c}}_{i},
$$

where $\overline{\mathbf{c}}_{i}, 1 \leq i \leq d$, and $\Pi$ are defined in (2.12).

Similarly, for $\mathbf{v} \in\left[L^{2}\left(\gamma_{i}^{s}\right)\right]^{m}, s=+,-, 1 \leq i \leq d$, we define

$$
\tilde{\pi}_{i}^{s} \mathbf{v}(\mathbf{x})=\Pi_{i}^{s} \mathbf{v}+\sum_{j \in D(i)} L_{p}\left(\frac{x_{i}}{h}\right) \operatorname{sgn}\left(\mathbf{B}_{i}\right) \overline{\mathbf{c}}_{i j}^{s},
$$

where $\overline{\mathbf{c}}_{i j}^{s}$ and $\Pi_{i}^{s}, s=+,-, j \in D(i), 1 \leq i \leq d$, are defined in (2.13a).

Lemma 3.2. Let $\mathbf{R}$ be defined in (3.2a), $\pi$ in (2.12), $\pi_{i}^{s}$ in (2.13a), $\tilde{\pi}$ in (3.8), and $\tilde{\pi}_{i}^{s}$ in (3.9), $s=+,-, 1 \leq i \leq d$. The following holds for any $\mathbf{v} \in\left[C^{p+2}(\bar{\omega})\right]^{m}$ :

$$
\begin{aligned}
& \mathbf{R}(\pi \mathbf{v})(\mathbf{x})=\tilde{\pi}(\mathbf{R v})(\mathbf{x}), \quad \mathbf{x} \in \omega, \text { and } \\
& \mathbf{R}\left(\pi_{i}^{s} \mathbf{v}\right)(\mathbf{x})=\tilde{\pi}_{i}^{s}(\mathbf{R v})(\mathbf{x}), \quad \mathbf{x} \in \gamma_{i}, \quad s=+,-, \quad 1 \leq i \leq d .
\end{aligned}
$$

Proof. By the definition of $\tilde{\pi}(\mathbf{R v})$ in (3.8) and the definition of $\overline{\mathbf{c}}_{i}$ in (2.12), we get

$$
\begin{aligned}
\tilde{\pi}(\mathbf{R v})(\mathbf{x}) & =\Pi(\mathbf{R v})(\mathbf{x})+\sum_{i=1}^{d} L_{p}\left(\frac{x_{i}}{h}\right) \operatorname{sgn}\left(\mathbf{B}_{i}\right) \mathbf{R} \overline{\mathbf{c}}_{i} \\
& =\mathbf{R}(\Pi \mathbf{v})(\mathbf{x})+\mathbf{R} \sum_{i=1}^{d} L_{p}\left(\frac{x_{i}}{h}\right) \operatorname{sgn}\left(\mathbf{A}_{i}\right) \overline{\mathbf{c}}_{i} \\
& =\mathbf{R}(\pi \mathbf{v})(\mathbf{x}),
\end{aligned}
$$


where we used (3.3) and the fact that the $L^{2}$ projection is commutable with any finite-dimensional linear operator.

The proof of (3.10b follows the same reasoning and is therefore omitted.

Substituting (3.10) and (3.5b-c) into (3.7) yields

$$
\begin{gathered}
\mathbf{U}_{h}(0, \mathbf{x})=\tilde{\pi} \mathbf{U}(0, \mathbf{x}), \quad \mathbf{x} \in \omega, \\
\left(\nu_{i} \mathbf{B}_{i}\right)^{-} \mathbf{U}_{h}^{-}(t, \mathbf{x})=\left(\nu_{i} \mathbf{B}_{i}\right)^{-} \tilde{\pi}_{i}^{s} \mathbf{U}^{-}(t, \mathbf{x}), \\
\mathbf{x} \in \gamma_{i}^{s}, s=+,-, 1 \leq i \leq d, 0<t<T .
\end{gathered}
$$

Therefore, $\mathbf{U}=\mathbf{R u}$ satisfies the symmetric system (3.5), while $\mathbf{U}_{h}=\mathbf{R u}_{h}$ satisfies the DG formulation (3.6) and (3.12). In the following section we apply the results established in 8 to $\mathbf{U}$ and $\mathbf{U}_{h}$.

3.1. Asymptotic behavior of the local discretization error. Now, we are ready to state a theorem for the local discretization error $\mathbf{e}=\mathbf{u}-\mathbf{u}_{h}$.

Theorem 3.3. Let $\mathbf{u} \in \mathfrak{C}^{p+2}$ and $\mathbf{u}_{h} \in \mathcal{P}_{p}$, respectively, be the solutions of (2.1) and (3.1). Then the local finite element error on $\omega$, at $t=\mathcal{O}(1)$ and $p \geq 1$, can be written as

$$
\mathbf{e}(t, h \boldsymbol{\xi})=\mathbf{u}(t, h \boldsymbol{\xi})-\mathbf{u}_{h}(t, h \boldsymbol{\xi})=h^{p+1} \sum_{i=1}^{d} \mathbf{r}_{i}\left(t, h \xi_{i}\right)+\mathcal{O}\left(h^{p+2}\right), \boldsymbol{\xi} \in \Delta,
$$

where

$$
\mathbf{r}_{i}\left(t, h \xi_{i}\right)=L_{p+1}\left(\xi_{i}\right) \mathbf{c}_{i}(t)-L_{p}\left(\xi_{i}\right)\left(\operatorname{sgn}\left(\mathbf{A}_{i}\right) \mathbf{c}_{i}(t)+\mathbf{d}_{i}(t)\right), 1 \leq i \leq d,
$$

with

$$
\mathbf{c}_{i}(t)=\frac{1}{a_{p+1}} \frac{1}{(p+1) !} \frac{\partial^{p+1} \mathbf{u}(t, \mathbf{0})}{\partial x_{i}^{p+1}}, \quad \mathbf{d}_{i}(t) \in \mathcal{N}\left(\mathbf{A}_{i}\right) \cap \bigoplus_{k=1}^{d} \mathcal{R}\left(\mathbf{A}_{k}\right),
$$

where $\bigoplus$ denotes the direct sum.

Proof. $\mathbf{U}=\mathbf{R u}$ satisfies the symmetric system (3.5), while $\mathbf{U}_{h}=\mathbf{R} \mathbf{u}_{h}$ satisfies the DG formulation (3.6) and (3.12). Since $\mathbf{u} \in \mathfrak{C}^{p+2}$ and $\mathbf{u}_{h} \in \mathcal{P}_{p}$, we have $\mathbf{U}=\mathbf{R u} \in \mathfrak{C}^{p+2}$ and $\mathbf{U}_{h}=\mathbf{R u}_{h} \in \mathcal{P}_{p}$. By Lemma 3.1. $\mathbf{B}_{i}$ is symmetric for $1 \leq i \leq d$. Thus, $\mathbf{U}$ and $\mathbf{U}_{h}$ satisfy the conditions of Theorem 3.6 in 8 . Therefore, for $p \geq 1$ and $t=\mathcal{O}(1)$,

$$
\mathbf{U}(t, h \boldsymbol{\xi})-\mathbf{U}_{h}(t, h \boldsymbol{\xi})=h^{p+1} \sum_{i=1}^{d} \tilde{\mathbf{r}}_{i}\left(t, h \xi_{i}\right)+\mathcal{O}\left(h^{p+2}\right), \boldsymbol{\xi} \in \Delta,
$$

where

$$
\tilde{\mathbf{r}}_{i}\left(t, h \xi_{i}\right)=L_{p+1}\left(\xi_{i}\right) \tilde{\mathbf{c}}_{i}(t)-L_{p}\left(\xi_{i}\right)\left(\operatorname{sgn}\left(\mathbf{B}_{i}\right) \tilde{\mathbf{c}}_{i}(t)+\tilde{\mathbf{d}}_{i}(t)\right), 1 \leq i \leq d,
$$

with

$$
\tilde{\mathbf{c}}_{i}(t)=\frac{1}{a_{p+1}} \frac{1}{(p+1) !} \frac{\partial^{p+1} \mathbf{U}(t, \mathbf{0})}{\partial x_{i}^{p+1}}, \quad \tilde{\mathbf{d}}_{i}(t) \in \mathcal{N}\left(\mathbf{B}_{i}\right) \cap \bigoplus_{k=1}^{d} \mathcal{R}\left(\mathbf{B}_{k}\right) .
$$

We obtain (3.13) by left-multiplying (3.14) by $\mathbf{R}^{-1}$ and substituting $\mathbf{u}=\mathbf{R}^{-1} \mathbf{U}$, $\mathbf{u}_{h}=\mathbf{R}^{-1} \mathbf{u}_{h}, \mathbf{r}_{i}=\mathbf{R}^{-1} \tilde{\mathbf{r}}_{i}, \mathbf{c}_{i}=\mathbf{R}^{-1} \tilde{\mathbf{c}}_{i}$ and $\mathbf{d}_{i}=\mathbf{R}^{-1} \tilde{\mathbf{d}}_{i}, 1 \leq i \leq d$. Furthermore, (3.4a) and (3.4b) lead to $\mathbf{d}_{i}=\mathbf{R}^{-1} \tilde{\mathbf{d}}_{i} \in \mathcal{N}\left(\mathbf{A}_{i}\right) \cap \bigoplus_{k=1}^{d} \mathcal{R}\left(\mathbf{A}_{k}\right)$. 
In order for the DG solution $\mathbf{u}_{h}$ to be $\mathcal{O}\left(h^{p+2}\right)$-superconvergent at any point in $\omega$, the leading error term shown in Theorem 3.3 has to be zero at this point. This pointwise superconvergence happens for nonsymmetric systems that satisfy the assumptions of the following theorem.

Since for nonsymmetric matrices, $\mathcal{R}\left(\mathbf{A}_{i}^{ \pm}\right) \neq \mathcal{R}\left(\left(\mathbf{A}_{i}^{ \pm}\right)^{t}\right)$, the superconvergence results stated in the following theorem are different from those in [8]. Here $\mathbf{z} \in$ $\bigcap_{i=1}^{d} \mathcal{R}\left(\left(\mathbf{A}_{i}^{s_{i}}\right)^{t}\right)$ instead of $\bigcap_{i=1}^{d} \mathcal{R}\left(\left(\mathbf{A}_{i}^{s_{i}}\right)\right), s_{i}=+,-$.

Theorem 3.4. Under the conditions of Theorem 3.3, with $p \geq 1$ and $t=\mathcal{O}(1)$, we let $\bar{\xi}_{j}^{s}, 1 \leq j \leq p+1$, denote the roots of $R_{p+1}^{s}(\xi), s=+,-$, shifted to $[0,1]$. Thus,

i) If there exists a unit vector $\mathbf{z} \in \bigcap_{i=1}^{d} \mathcal{R}\left(\left(\mathbf{A}_{i}^{s_{i}}\right)^{t}\right), s_{i}=+,-$, then the projection $\mathbf{z}^{t} \mathbf{e}(t, \mathbf{x})$ of the local error onto span $\{\mathbf{z}\}$ is $\mathcal{O}\left(h^{p+2}\right)$ superconvergent at the points $(t, h \overline{\boldsymbol{\xi}}), \bar{\xi}_{i}=\bar{\xi}_{k_{i}}^{s_{i}}, 1 \leq k_{i} \leq p+1,1 \leq i \leq d$, i.e.,

$$
\mathbf{z}^{t} \mathbf{e}(t, h \overline{\boldsymbol{\xi}})=\mathcal{O}\left(h^{p+2}\right) \text {. }
$$

ii) Moreover, if $\gamma_{i}(a)=\left\{\mathbf{x} \in(0, h)^{d}: x_{i}=a\right\}, 0 \leq a \leq h$, and if $\mathbf{v} \in \mathcal{P}_{p-1}$ is a unit vector with respect to the $C^{\infty}$ norm, then, at $a=h \bar{\xi}_{k}^{s}$, we have the superconvergence of the following error averages:

$$
\frac{1}{h^{d-1}} \int_{\gamma_{i}\left(h \bar{\xi}_{k}^{s}\right)} \mathbf{v}^{t} \mathbf{A}_{i}^{s} \mathbf{e} d \mathbf{s}=\mathcal{O}\left(h^{p+2}\right), 1 \leq k \leq p+1, s=+,-, 1 \leq i \leq d
$$

and

$$
\frac{1}{h^{d-1}} \int_{\gamma_{i}^{s}} \mathbf{v}^{t}\left(\left(\nu_{i} \mathbf{A}_{i}\right)^{+} \mathbf{e}+\left(\nu_{i} \mathbf{A}_{i}\right)^{-} \mathbf{e}^{-}\right) d \mathbf{s}=\mathcal{O}\left(h^{p+2}\right), \quad s=+,-, \quad 1 \leq i \leq d .
$$

Proof. We will prove (3.15) for the case $s_{i}=+, 1 \leq i \leq d$.

Thus, assume that there exists a unit vector $\mathbf{z} \in \bigcap_{i=1}^{d} \mathcal{R}\left(\left(\mathbf{A}_{i}^{+}\right)^{t}\right)$, i.e., there exists $\mathbf{v}_{i}$ such that

$$
\left(\mathbf{A}_{i}^{+}\right)^{t} \mathbf{v}_{i}=\mathbf{z}, 1 \leq i \leq d
$$

Left pre-multiplying e in (3.13a) by $\mathbf{z}^{t}$ and evaluating the resulting function at the points $(t, h \overline{\boldsymbol{\xi}}), \overline{\boldsymbol{\xi}}=\left(\bar{\xi}_{k_{1}}^{+}, \ldots, \bar{\xi}_{k_{d}}^{+}\right), 1 \leq k_{i} \leq p+1,1 \leq i \leq d$, we obtain

$$
\mathbf{z}^{t} \mathbf{e}(t, h \overline{\boldsymbol{\xi}})=h^{p+1} \sum_{i=1}^{d}\left(L_{p+1}\left(\bar{\xi}_{k_{i}}^{+}\right) \mathbf{z}^{t} \mathbf{c}_{i}-L_{p}\left(\bar{\xi}_{k_{i}}^{+}\right)\left(\mathbf{z}^{t} \operatorname{sgn}\left(\mathbf{A}_{i}\right) \mathbf{c}_{i}+\mathbf{z}^{t} \mathbf{d}_{i}\right)\right)+\mathcal{O}\left(h^{p+2}\right) .
$$

By the property (2.3b) and (3.13c) we have $\mathbf{d}_{i} \in \mathcal{N}\left(\mathbf{A}_{i}\right) \subseteq \mathcal{N}\left(\mathbf{A}_{i}^{+}\right)$, which yields by (3.18)

$$
\mathbf{z}^{t} \mathbf{d}_{i}=\mathbf{v}_{i}^{t} \mathbf{A}_{i}^{+} \mathbf{d}_{i}=\mathbf{0} .
$$

Applying (3.18) and the property (2.3d) yields

$$
\mathbf{z}^{t} \operatorname{sgn}\left(\mathbf{A}_{i}\right) \mathbf{c}_{i}=\mathbf{v}_{i}^{t} \mathbf{A}_{i}^{+} \operatorname{sgn}\left(\mathbf{A}_{i}\right) \mathbf{c}_{i}=\mathbf{v}_{i}^{t} \mathbf{A}_{i}^{+} \mathbf{c}_{i}=\mathbf{z}^{t} \mathbf{c}_{i} .
$$

Substituting (3.20) and (3.21) into (3.19), we prove that

$$
\mathbf{z}^{t} \mathbf{e}(t, h \overline{\boldsymbol{\xi}})=h^{p+1} \sum_{i=1}^{d} R_{p+1}^{+}\left(\bar{\xi}_{k_{i}}^{+}\right) \mathbf{z}^{t} \mathbf{c}_{i}+\mathcal{O}\left(h^{p+2}\right)=\mathcal{O}\left(h^{p+2}\right) .
$$

Following the same line of reasoning we establish 3.15 for all other cases.

The proof of (3.16) and (3.17) can be found in 9] for symmetric systems and $d=2$ and is therefore omitted. 


\section{A Posteriori error estimation}

Here we present an a posteriori error estimation procedure for symmetrizable systems using projections onto the range and null spaces of the coefficient matrices $\mathbf{A}_{i}, 1 \leq i \leq d$ to split the DG error into two parts as explained below.

Let us consider a symmetrizable matrix $\mathbf{M}$ with real eigenpairs $\left(\lambda_{j}, \mathbf{p}_{j}\right), j=$ $1, \ldots, m$ such that $\lambda_{j} \neq 0, j=1, \ldots, r$ and $\lambda_{r+1}=\cdots=\lambda_{m}=0$. Thus, $\mathcal{R}(M)=$ $\operatorname{span}\left(\mathbf{p}_{1}, \ldots, \mathbf{p}_{r}\right)$ and $\mathcal{N}(M)=\operatorname{span}\left(\mathbf{p}_{r+1}, \ldots, \mathbf{p}_{m}\right)$. Thus, every $\mathbf{v} \in \mathbb{R}^{m}$ can be written as

$$
\mathbf{v}=\mathbf{v}^{\mathcal{R}}+\mathbf{v}^{\mathcal{N}}, \text { where } \mathbf{v}^{\mathcal{R}} \in \mathcal{R}(M), \mathbf{v}^{\mathcal{N}} \in \mathcal{N}(M) .
$$

Thus, for each matrix $\mathbf{M}=\mathbf{A}_{i}$ we have the following projections:

$$
\mathrm{P}_{i}^{\mathcal{R}} \mathbf{v}=\mathbf{v}^{\mathcal{R}} \text { and } \mathrm{P}_{i}^{\mathcal{N}}=I-\mathrm{P}_{i}^{\mathcal{R}} .
$$

Next, the Drazin inverse [26] of a symmetrizable matrix $\mathbf{M}=\mathbf{P} \operatorname{diag}\left(\lambda_{1}, \ldots, \lambda_{m}\right) \mathbf{P}^{-1}$ is defined by

$$
\mathbf{M}^{D}=\mathbf{P} \operatorname{diag}\left(\lambda_{1}^{\dagger}, \ldots, \lambda_{m}^{\dagger}\right) \mathbf{P}^{-1}
$$

where

$$
x^{\dagger}= \begin{cases}x^{-1}, & \text { if } x \neq 0, \\ 0, & \text { if } x=0 .\end{cases}
$$

From the definition of the Drazin inverse for symmetrizable matrices we have

$$
\left(\mathbf{M}^{D}\right)^{t}=\left(\mathbf{M}^{t}\right)^{D} .
$$

In the following lemma we show that the projections $\mathrm{P}_{i}^{\mathcal{R}}$ and $\mathrm{P}_{i}^{\mathcal{N}}$ corresponding to a symmetrizable matrix $\mathbf{A}_{i}$ can be expressed in terms of the Drazin inverse $\mathbf{A}_{i}^{D}$.

Lemma 4.1. Let $\mathbf{A}_{i}^{D}$ be the Drazin inverse of $\mathbf{A}_{i}, 1 \leq i \leq d$. Then,

i) $\mathrm{P}_{i}^{\mathcal{R}}=\mathbf{A}_{i}^{D} \mathbf{A}_{i}=\mathbf{A}_{i} \mathbf{A}_{i}^{D}$,

ii) $\mathrm{P}_{i}^{\mathcal{N}}=\mathbf{I}-\mathbf{A}_{i}^{D} \mathbf{A}_{i}$.

Proof. The proof is established by direct verification.

In the next lemma we show that the leading term of the discretization error may be split into two components.

Lemma 4.2. Under the assumptions of Theorem 3.3, for $p>1$ and $t=\mathcal{O}(1)$, the leading term of the spatial discretization error can be split into two parts as

$$
\mathbf{e}=\mathbf{e}^{\mathcal{R}}+\mathbf{e}^{\mathcal{N}}+\mathcal{O}\left(h^{p+2}\right),
$$

where

$$
\begin{aligned}
& \mathbf{e}^{\mathcal{R}}(t, h \boldsymbol{\xi})=h^{p+1} \sum_{i=1}^{d}\left(L_{p+1}\left(\xi_{i}\right) \mathbf{c}_{i}^{\mathcal{R}}(t)-L_{p}\left(\xi_{i}\right) \operatorname{sgn}\left(\mathbf{A}_{i}\right) \mathbf{c}_{i}^{\mathcal{R}}(t)\right), \\
& \mathbf{e}^{\mathcal{N}}(t, h \boldsymbol{\xi})=h^{p+1} \sum_{i=1}^{d}\left(L_{p+1}\left(\xi_{i}\right) \mathbf{c}_{i}^{\mathcal{N}}(t)-L_{p}\left(\xi_{i}\right) \mathbf{d}_{i}(t)\right),
\end{aligned}
$$

with

$$
\mathbf{c}_{i}^{\mathcal{R}}=\mathrm{P}_{i}^{\mathcal{R}} \mathbf{c}_{i} \in \mathcal{R}\left(\mathbf{A}_{i}\right), \quad \mathbf{c}_{i}^{\mathcal{N}}=\mathrm{P}_{i}^{\mathcal{N}} \mathbf{c}_{i}, \mathbf{d}_{i} \in \mathcal{N}\left(\mathbf{A}_{i}\right), \quad 1 \leq i \leq d .
$$


Proof. Under the assumptions of Theorem 3.3, the local discretization error for the DG method on $\omega=(0, h)^{d}$, for $p>1$ and $t=\mathcal{O}(1)$, can be written as

$$
\mathbf{e}(t, h \boldsymbol{\xi})=h^{p+1} \sum_{i=1}^{d}\left(L_{p+1}\left(\xi_{i}\right) \mathbf{c}_{i}(t)-L_{p}\left(\xi_{i}\right)\left(\operatorname{sgn}\left(\mathbf{A}_{i}\right) \mathbf{c}_{i}(t)+\mathbf{d}_{i}(t)\right)\right)+\mathcal{O}\left(h^{p+2}\right),
$$

where $\mathbf{d}_{i}(t) \in \mathcal{N}\left(\mathbf{A}_{i}\right)$. Applying Lemma 4.1 to (4.6) yields (4.5).

We note that if all matrices $\mathbf{A}_{i}, 1 \leq i \leq d$, are invertible, the error component $\mathbf{e}^{\mathcal{N}}(t, \mathbf{x})$ is zero. (3.5).

Lemma 4.3. Under the assumptions of Theorem 3.3. for $p>1$ and $t=\mathcal{O}(1)$, let $\mathbf{U}=\mathbf{R u}, \mathbf{U}_{h}=\mathbf{R u}_{h}, \tilde{\mathbf{e}}^{\mathcal{N}}=\mathbf{R} \mathbf{e}^{\mathcal{N}}, \tilde{\mathbf{e}}^{\mathcal{R}}=\mathbf{R e}^{\mathcal{R}}, \tilde{\mathbf{e}}^{\mathcal{N}}=\mathbf{R} \mathbf{e}^{\mathcal{N}}, \tilde{\mathbf{c}}_{i}^{\mathcal{R}}=\mathbf{R c}_{i}^{\mathcal{R}}$, $\tilde{\mathbf{c}}_{i}^{\mathcal{N}}=\mathbf{R} \mathbf{c}_{i}^{\mathcal{N}}$, and $\tilde{\mathbf{d}}_{i}=\mathbf{R d}_{i}$. Then, the leading term of the spatial discretization error for the symmetrized systems (3.5) and (3.6) can be split into two parts as

$$
\mathbf{U}-\mathbf{U}_{h}=\tilde{\mathbf{e}}^{\mathcal{R}}+\tilde{\mathbf{e}}^{\mathcal{N}}+\mathcal{O}\left(h^{p+2}\right),
$$

where

$$
\begin{aligned}
& \tilde{\mathbf{e}}^{\mathcal{R}}(t, h \boldsymbol{\xi})=h^{p+1} \sum_{i=1}^{d}\left(L_{p+1}\left(\xi_{i}\right) \tilde{\mathbf{c}}_{i}^{\mathcal{R}}(t)-L_{p}\left(\xi_{i}\right) \operatorname{sgn}\left(\mathbf{B}_{i}\right) \tilde{\mathbf{c}}_{i}^{\mathcal{R}}(t)\right), \\
& \mathbf{e}^{\mathcal{N}}(t, h \boldsymbol{\xi})=h^{p+1} \sum_{i=1}^{d}\left(L_{p+1}\left(\xi_{i}\right) \tilde{\mathbf{c}}_{i}^{\mathcal{N}}(t)-L_{p}\left(\xi_{i}\right) \tilde{\mathbf{d}}_{i}(t)\right),
\end{aligned}
$$

with

$$
\tilde{\mathbf{c}}_{i}^{\mathcal{R}} \in \mathcal{R}\left(\mathbf{B}_{i}\right), \quad \tilde{\mathbf{c}}_{i}^{\mathcal{N}}, \tilde{\mathbf{d}}_{i} \in \mathcal{N}\left(\mathbf{B}_{i}\right), \quad 1 \leq i \leq d .
$$

Proof. First, we left-multiply (4.5)-c) by $\mathbf{R}$ and substitute $\mathbf{U}=\mathbf{R u}, \mathbf{U}_{h}=\mathbf{R} \mathbf{u}_{h}$, $\tilde{\mathbf{e}}^{\mathcal{N}}=\mathbf{R} \mathbf{e}^{\mathcal{N}}, \tilde{\mathbf{e}}^{\mathcal{R}}=\mathbf{R e}^{\mathcal{R}}, \tilde{\mathbf{e}}^{\mathcal{N}}=\mathbf{R e}^{\mathcal{N}}, \tilde{\mathbf{c}}_{i}^{\mathcal{R}}=\mathbf{R c}_{i}^{\mathcal{R}}, \tilde{\mathbf{c}}_{i}^{\mathcal{N}}=\mathbf{R c}_{i}^{\mathcal{N}}$, and $\tilde{\mathbf{d}}_{i}=\mathbf{R d}_{i}$.

Applying

$$
\mathbf{R} \operatorname{sgn}\left(\mathbf{A}_{i}\right)=\operatorname{sgn}\left(\mathbf{B}_{i}\right) \mathbf{R},
$$

which is obtained from (3.2b), yields (4.7 $\mathrm{a}-\mathrm{c})$.

Finally, combining (4.5d) and Lemma 3.1 leads to (4.7d).

Next, we describe an a posteriori error estimation procedure for estimating both $\mathbf{e}^{\mathcal{R}}$ and $\mathbf{e}^{\mathcal{N}}$ (when needed). We prove that, for smooth solutions, our local error estimates converge to the true error under mesh refinement. Up to this point we are not able to prove the asymptotic exactness of our global a posteriori error estimates. However, computational results for several hyperbolic systems shown in 5 suggest that our global a posteriori error estimates are asymptotically exact for smooth solutions.

Since the range and null space are not orthogonal for nonsymmetric matrices, we need to define test function spaces for the error estimation that are different from the solution space. Thus, we solve local Petrov-Galerkin problems in order to compute error estimates. 
4.1. The stationary error estimate. The stationary a posteriori error estimation procedure for computing estimates for $\mathbf{e}^{\mathcal{R}}$ consists of determining

(4.8a)

$$
\mathbf{E}^{\mathcal{R}}(t, h \boldsymbol{\xi})=\sum_{j=1}^{d}\left(L_{p+1}\left(\xi_{j}\right)-L_{p}\left(\xi_{j}\right) \operatorname{sgn}\left(\mathbf{A}_{j}\right)\right) \boldsymbol{\gamma}_{j}^{\mathcal{R}}(t), \boldsymbol{\gamma}_{j}^{\mathcal{R}} \in \mathcal{R}\left(\mathbf{A}_{j}\right), 1 \leq j \leq d,
$$

such that

$\int_{\omega} L_{p}\left(\frac{x_{i}}{h}\right) \mathbf{v}^{t}\left(\frac{\partial \mathbf{u}_{h}}{\partial t}+\sum_{j=1}^{d} \mathbf{A}_{j} \frac{\partial\left(\mathbf{u}_{h}+\mathbf{E}^{\mathcal{R}}\right)}{\partial x_{j}}-\mathbf{g}\right) d \mathbf{x}=0, \forall \mathbf{v} \in \mathcal{R}\left(\mathbf{A}_{i}^{t}\right), 1 \leq i \leq d$.

By Lemma 4.1 and (4.4), $\left(\mathrm{P}_{i}^{\mathcal{R}}\right)^{t}=\left(\mathbf{A}_{i}^{D} \mathbf{A}_{i}\right)^{t}=\left(\mathbf{A}_{i}^{t}\right)^{D} \mathbf{A}_{i}^{t}$ is the projection onto $\mathcal{R}\left(\mathbf{A}_{i}^{t}\right)$, thus $\mathcal{R}\left(\left(\mathrm{P}_{i}^{\mathcal{R}}\right)^{t}\right)=\mathcal{R}\left(\mathbf{A}_{i}^{t}\right)$.

Since (4.8b) holds for all $\mathbf{v} \in \mathcal{R}\left(\mathbf{A}_{i}^{t}\right)$ and the columns of $\left(\mathrm{P}_{i}^{\mathcal{R}}\right)^{t}$ are in the range of $\mathbf{A}_{i}^{t}$, thus (4.8b) yields

$$
\int_{\omega} L_{p}\left(\frac{x_{i}}{h}\right) \mathbf{P}_{i}^{\mathcal{R}}\left(\frac{\partial \mathbf{u}_{h}}{\partial t}+\sum_{j=1}^{d} \mathbf{A}_{j} \frac{\partial\left(\mathbf{u}_{h}+\mathbf{E}^{\mathcal{R}}\right)}{\partial x_{j}}-\mathbf{g}\right) d \mathbf{x}=\mathbf{0}, 1 \leq i \leq d .
$$

Substituting (4.8a) into (4.9) and applying the orthogonality properties (2.11), we obtain

$$
\mathbf{A}_{i} \gamma_{i}^{\mathcal{R}} \int_{\omega} L_{p}\left(\frac{x_{i}}{h}\right) L_{p+1}^{\prime}\left(\frac{x_{i}}{h}\right) d \mathbf{x}=\mathfrak{r}_{p, i}^{\mathcal{R}}
$$

where $\mathfrak{r}_{p, i}^{\mathcal{R}}$ is the projection of the residual onto $\mathcal{R}\left(\mathbf{A}_{i}\right)$ as

$$
\mathfrak{r}_{p, i}^{\mathcal{R}}=\mathrm{P}_{i}^{\mathcal{R}} \int_{\omega} L_{p}\left(\frac{x_{i}}{h}\right)\left(\mathbf{g}-\frac{\partial \mathbf{u}_{h}}{\partial t}-\sum_{j=1}^{d} \mathbf{A}_{j} \frac{\partial \mathbf{u}_{h}}{\partial x_{j}}\right) d \mathbf{x}, \quad 1 \leq i \leq d .
$$

Using (2.11) we further reduce 4.10a), obtaining

$$
2 h^{d-1} \mathbf{A}_{i} \gamma_{i}^{\mathcal{R}}=\mathfrak{r}_{p, i}^{\mathcal{R}}, \quad 1 \leq i \leq d .
$$

Since $\mathfrak{r}_{p, i}^{\mathcal{R}} \in \mathcal{R}\left(\mathbf{A}_{i}\right)$, we can solve (4.11) to find the unique solution $\gamma_{i}^{\mathcal{R}} \in \mathcal{R}\left(\mathbf{A}_{i}\right)$,

$$
\gamma_{i}^{\mathcal{R}}=\frac{h^{1-d}}{2} \mathbf{A}_{i}^{D} \mathfrak{r}_{p, i}^{\mathcal{R}}, \quad 1 \leq i \leq d .
$$

Next, we show that this stationary error estimate is asymptotically exact under mesh refinement.

Theorem 4.4. Under the assumptions of Theorem 3.3, let us consider the error estimate

$$
\mathbf{E}^{\mathcal{R}}(t, h \boldsymbol{\xi})=\sum_{i=1}^{d}\left(L_{p+1}\left(\xi_{i}\right)-L_{p}\left(\xi_{i}\right) \operatorname{sgn}\left(\mathbf{A}_{i}\right)\right) \frac{h^{1-d}}{2} \mathbf{A}_{i}^{D} \mathfrak{r}_{p, i}^{\mathcal{R}},
$$

where $\mathfrak{r}_{p, i}^{\mathcal{R}}, 1 \leq i \leq d$, are defined in (4.10b).

Then, for $p \geq 1$ and $t=\mathcal{O}(1)$,

$$
\mathbf{e}^{\mathcal{R}}(t, \mathbf{x})=\mathbf{E}^{\mathcal{R}}(t, \mathbf{x})+\mathcal{O}\left(h^{p+2}\right), \quad \mathbf{x} \in \omega .
$$


Proof. Left-multiplying (4.8a) by $\mathbf{R}$ and substituting $\tilde{\mathbf{E}}^{\mathcal{R}}=\mathbf{R} \mathbf{E}^{\mathcal{R}}$ and $\tilde{\boldsymbol{\gamma}}_{i}^{\mathcal{R}}=\mathbf{R} \boldsymbol{\gamma}_{i}^{\mathcal{R}}$ yields

$$
\tilde{\mathbf{E}}^{\mathcal{R}}(t, h \boldsymbol{\xi})=\sum_{j=1}^{d}\left(L_{p+1}\left(\xi_{j}\right)-L_{p}\left(\xi_{j}\right) \operatorname{sgn}\left(\mathbf{B}_{j}\right)\right) \tilde{\gamma}_{j}^{\mathcal{R}}(t), \tilde{\gamma}_{j}^{\mathcal{R}} \in \mathcal{R}\left(\mathbf{B}_{j}\right), 1 \leq j \leq d,
$$

where we used (3.4b) and the fact that $\gamma_{j}^{\mathcal{R}} \in \mathcal{R}\left(\mathbf{A}_{j}\right)$.

Substituting $\mathbf{v}=\mathbf{R}^{t} \mathbf{w}, \mathbf{U}_{h}=\mathbf{R} \mathbf{u}_{h}$, and $\tilde{\mathbf{E}}^{\mathcal{R}}=\mathbf{R} \mathbf{E}^{\mathcal{R}}$ into (4.8b) yields

$$
\begin{array}{r}
\int_{\omega} L_{p}\left(\frac{x_{i}}{h}\right) \mathbf{w}^{t}\left(\frac{\partial \mathbf{U}_{h}}{\partial t}+\sum_{j=1}^{d} \mathbf{B}_{j} \frac{\partial\left(\mathbf{U}_{h}+\tilde{\mathbf{E}}^{\mathcal{R}}\right)}{\partial x_{j}}-\mathbf{R g}\right) d \mathbf{x}=0, \\
\forall \mathbf{w} \in \mathcal{R}\left(\mathbf{B}_{i}\right), 1 \leq i \leq d,
\end{array}
$$

where we used (3.4d) and the fact that $\mathbf{v} \in \mathcal{R}\left(\mathbf{A}_{i}^{t}\right)$.

Since system (4.15) satisfies Theorem 4.1 in [8], we obtain, for $p \geq 1$ and $t=$ $\mathcal{O}(1)$

$$
\tilde{\mathbf{e}}^{\mathcal{R}}(t, \mathbf{x})=\tilde{\mathbf{E}}^{\mathcal{R}}(t, \mathbf{x})+\mathcal{O}\left(h^{p+2}\right), \quad \mathbf{x} \in \omega .
$$

Left-multiplying (4.16) with $\mathbf{R}^{-1}$ and substituting $\mathbf{e}^{\mathcal{R}}=\mathbf{R}^{-1} \tilde{\mathbf{e}}^{\mathcal{R}}$ and $\mathbf{E}^{\mathcal{R}}=\mathbf{R}^{-1} \tilde{\mathbf{E}}^{\mathcal{R}}$ yields (4.14).

4.2. The transient error estimate. Here we present a transient a posteriori error estimation procedure to compute estimates for $\mathbf{e}^{\mathcal{N}}$. Note that if all $\mathbf{A}_{i}, 1 \leq i \leq d$, are invertible, $\mathbf{e}^{\mathcal{N}}=\mathbf{0}$ by definition in (4.5c).

By Lemma 2.2 the approximations $\pi \mathbf{u}_{0}$ on $\omega$ and $\pi_{i}^{s} \mathbf{u}$ on the boundary $\partial \omega$ satisfy

$$
\begin{aligned}
\mathbf{e}(0, \mathbf{x}) & =\mathbf{u}_{0}(\mathbf{x})-\pi \mathbf{u}_{0}(\mathbf{x}) \\
(4.17) \quad & =h^{p+1} \sum_{j=1}^{d}\left(L_{p+1}\left(\frac{x_{j}}{h}\right) \mathbf{c}_{j}(0)-L_{p}\left(\frac{x_{j}}{h}\right) \operatorname{sgn}\left(\mathbf{A}_{j}\right) \mathbf{c}_{j}(0)\right)+\mathcal{O}\left(h^{p+2}\right), \mathbf{x} \in \omega, \\
\mathbf{e}^{-}(t, \mathbf{x}) & =\mathbf{u}(t, \mathbf{x})-\pi_{i}^{s} \mathbf{u}(t, \mathbf{x}) \\
& =h^{p+1} \sum_{j \in D(i)}\left(L_{p+1}\left(\frac{x_{j}}{h}\right) \mathbf{c}_{j}(t)-L_{p}\left(\frac{x_{j}}{h}\right) \operatorname{sgn}\left(\mathbf{A}_{j}\right) \mathbf{c}_{j}(t)\right)+\mathcal{O}\left(h^{p+2}\right), \\
(4.18) \quad \mathbf{x} & \in \gamma_{i}^{s}, \quad s=+,-, 1 \leq i \leq d .
\end{aligned}
$$

We split the error at $t=0$ into $\mathbf{e}=\mathbf{e}^{\mathcal{R}}+\mathbf{e}^{\mathcal{N}}+\mathcal{O}\left(h^{p+2}\right)$ as in 4.5a and define $\mathbf{E}^{\mathcal{N}}(0, \mathbf{x})$ by

$$
\mathbf{E}^{\mathcal{N}}(0, \mathbf{x})=\mathbf{e}^{\mathcal{N}}(0, \mathbf{x})=h^{p+1} \sum_{i=1}^{d}\left(L_{p+1}\left(\frac{x_{i}}{h}\right) \mathrm{P}_{i}^{\mathcal{N}} \overline{\mathbf{c}}_{i}(0)\right),
$$

where $\mathrm{P}_{i}^{\mathcal{N}} \overline{\mathbf{c}}_{i}(0)$ is the projection of $\overline{\mathbf{c}}_{i}(0)$ into $\mathcal{N}\left(\mathbf{A}_{i}\right)$.

On the boundary, we define $\mathbf{E}^{-}$by the leading term of (4.18),

$$
\begin{gathered}
\mathbf{E}^{-}(t, \mathbf{x})=h^{p+1} \sum_{j \in D(i)}\left(L_{p+1}\left(\frac{x_{j}}{h}\right) \overline{\mathbf{c}}_{i j}^{s}(t)-L_{p}\left(\frac{x_{j}}{h}\right) \operatorname{sgn}\left(\mathbf{A}_{j}\right) \overline{\mathbf{c}}_{i j}^{s}(t)\right), \\
\mathbf{x} \in \gamma_{i}^{s}, s=+,-, 1 \leq i \leq d .
\end{gathered}
$$


Then we define the error estimate for $\mathbf{e}^{\mathcal{N}}$ by determining the coefficients of (4.21a)

$\mathbf{E}^{\mathcal{N}}(t, \mathbf{x})=\sum_{j=1}^{d}\left(L_{p+1}\left(\frac{x_{j}}{h}\right) \boldsymbol{\gamma}_{j}^{\mathcal{N}}(t)-L_{p}\left(\frac{x_{j}}{h}\right) \boldsymbol{\delta}_{j}(t)\right), \quad \boldsymbol{\gamma}_{j}^{\mathcal{N}}, \boldsymbol{\delta}_{j} \in \mathcal{N}\left(\mathbf{A}_{j}\right), 1 \leq j \leq d$, such that

$$
\begin{aligned}
& \int_{\omega} \mathbf{v}^{t}\left(\frac{\partial\left(\mathbf{u}_{h}+\mathbf{E}^{\mathcal{N}}\right)}{\partial t}+\sum_{j=1}^{d} \mathbf{A}_{j} \frac{\partial \mathbf{u}_{h}}{\partial x_{j}}-\mathbf{g}\right) d \mathbf{x} \\
& =\sum_{j=1}^{d} \int_{\gamma_{j}} \mathbf{v}^{t}\left(\nu_{j} \mathbf{A}_{j}\right)^{-}\left(\mathbf{u}_{h}+\mathbf{E}^{\mathcal{R}}+\mathbf{E}^{\mathcal{N}}-\mathbf{u}_{h}^{-}-\mathbf{E}^{-}\right) d \mathbf{s}, \forall \mathbf{v} \in \mathcal{E}_{p},
\end{aligned}
$$

where $\mathbf{E}^{\mathcal{R}}$ equals the stationary component defined by (4.8) and

$$
\mathcal{E}_{p}=\left\{\mathbf{v}(\mathbf{x})=\sum_{i=1}^{d}\left(L_{p+1}\left(\frac{x_{i}}{h}\right) \mathbf{a}_{i}-L_{p}\left(\frac{x_{i}}{h}\right) \mathbf{b}_{i}\right): \mathbf{a}_{i}, \mathbf{b}_{i} \in \mathcal{N}\left(\mathbf{A}_{i}^{t}\right)\right\} .
$$

Thus, by Lemma 4.1 and (4.4), $\left(\mathrm{P}_{i}^{\mathcal{N}}\right)^{t}=\mathbf{I}-\left(\mathbf{A}_{i}^{D} \mathbf{A}_{i}\right)^{t}=\mathbf{I}-\left(\mathbf{A}_{i}^{t}\right)^{D} \mathbf{A}_{i}^{t}$ is the projection onto $\mathcal{N}\left(\mathbf{A}_{i}^{t}\right)$, and therefore $\mathcal{R}\left(\left(\mathrm{P}_{i}^{\mathcal{N}}\right)^{t}\right)=\mathcal{N}\left(\mathbf{A}_{i}^{t}\right)$.

Replacing $\mathbf{v}$ in (4.21b) by $L_{m}\left(\xi_{i}\right)\left(\mathrm{P}_{i}^{\mathcal{N}}\right)^{t}, m=p, p+1,1 \leq i \leq d$, yields

$$
\begin{aligned}
& \int_{\omega} L_{m}\left(\frac{x_{i}}{h}\right) \mathrm{P}_{i}^{\mathcal{N}}\left(\frac{\partial\left(\mathbf{u}_{h}+\mathbf{E}^{\mathcal{N}}\right)}{\partial t}+\sum_{j=1}^{d} \mathbf{A}_{j} \frac{\partial \mathbf{u}_{h}}{\partial x_{j}}-\mathbf{g}\right) d \mathbf{x} \\
&=\sum_{j=1}^{d} \int_{\gamma_{j}} L_{m}\left(\frac{x_{i}}{h}\right) \mathrm{P}_{i}^{\mathcal{N}}\left(\nu_{j} \mathbf{A}_{j}\right)^{-}\left(\mathbf{u}_{h}+\mathbf{E}^{\mathcal{R}}+\mathbf{E}^{\mathcal{N}}-\mathbf{u}_{h}^{-}-\mathbf{E}^{-}\right) d \mathbf{s}, \\
& \forall m=p, p+1,1 \leq i \leq d .
\end{aligned}
$$

By (2.3C), $\mathrm{P}_{i}^{\mathcal{N}}\left(\nu_{i} \mathbf{A}_{i}\right)^{-}=\mathbf{0}$, thus (4.22) can be written as

$$
\int_{\omega} L_{m}\left(\frac{x_{i}}{h}\right) \mathrm{P}_{i}^{\mathcal{N}} \frac{\partial \mathbf{E}^{\mathcal{N}}}{\partial t} d \mathbf{x}-\sum_{j \in D(i)} \int_{\gamma_{j}} L_{m}\left(\frac{x_{i}}{h}\right) \mathrm{P}_{i}^{\mathcal{N}}\left(\nu_{j} \mathbf{A}_{j}\right)^{-} \mathbf{E}^{\mathcal{N}} d \mathbf{s}=\mathfrak{r}_{m, i}^{\mathcal{N}},
$$

where $\mathfrak{r}_{m, i}^{\mathcal{N}}$ is the projection of the residual onto $\mathcal{N}\left(\mathbf{A}_{i}\right)$ along $\mathcal{R}\left(\mathbf{A}_{i}\right)$, given by

$$
\begin{aligned}
\mathfrak{r}_{m, i}^{\mathcal{N}} & =\mathrm{P}_{i}^{\mathcal{N}} \int_{\omega} L_{m}\left(\frac{x_{i}}{h}\right)\left(\mathbf{g}-\frac{\partial \mathbf{u}_{h}}{\partial t}-\sum_{j=1}^{d} \mathbf{A}_{j} \frac{\partial \mathbf{u}_{h}}{\partial x_{j}}\right) d \mathbf{x} \\
& \left.+\mathrm{P}_{i}^{\mathcal{N}} \sum_{j=1}^{d} \int_{\gamma_{j}} L_{m}\left(\frac{x_{i}}{h}\right)\left(\nu_{j} \mathbf{A}_{j}\right)^{-}\left(\mathbf{u}_{h}+\mathbf{E}^{\mathcal{R}}-\mathbf{u}_{h}^{-}-\mathbf{E}^{-}\right)\right) d \mathbf{s}, \\
& m=p, p+1,1 \leq i \leq d .
\end{aligned}
$$

For $m=p+1$, we use the orthogonality properties (2.11) to reduce (4.23a) to

$$
\int_{\omega} L_{p+1}^{2}\left(\frac{x_{i}}{h}\right) \dot{\gamma}_{i}^{\mathcal{N}} d \mathbf{x}-\sum_{j \in D(i)} \int_{\gamma_{j}} L_{p+1}^{2}\left(\frac{x_{i}}{h}\right) \mathrm{P}_{i}^{\mathcal{N}}\left(\nu_{j} \mathbf{A}_{j}\right)^{-} \gamma_{i}^{\mathcal{N}} d \mathbf{s}=\mathfrak{r}_{p+1, i}^{\mathcal{N}}
$$


which by (2.11) is equal to

$$
\dot{\gamma}_{i}^{\mathcal{N}}=\frac{1}{h} \mathrm{P}_{i}^{\mathcal{N}} \sum_{j \in D(i)}\left(\mathbf{A}_{j}^{-}-\mathbf{A}_{j}^{+}\right) \boldsymbol{\gamma}_{i}^{\mathcal{N}}+\frac{2 p+3}{h^{d}} \mathfrak{r}_{p+1, i}^{\mathcal{N}} .
$$

For $m=p$, similarly we get

$$
\dot{\boldsymbol{\delta}}_{i}=\frac{1}{h} \mathrm{P}_{i}^{\mathcal{N}} \sum_{j \in D(i)}\left(\mathbf{A}_{j}^{-}-\mathbf{A}_{j}^{+}\right) \boldsymbol{\delta}_{i}+\frac{2 p+1}{h^{d}} \mathfrak{r}_{p, i}^{\mathcal{N}},
$$

subject to the initial conditions

$$
\gamma_{i}^{\mathcal{N}}(0)=h^{p+1} \mathrm{P}_{i}^{\mathcal{N}} \overline{\mathbf{c}}_{i}(0), \boldsymbol{\delta}_{i}(0)=\mathbf{0} .
$$

Note that (4.25) and (4.23b) ensures that $\boldsymbol{\gamma}_{i}^{\mathcal{N}}, \boldsymbol{\delta}_{i} \in \mathcal{N}\left(\mathbf{A}_{i}\right), 1 \leq i \leq d$.

Then (4.25) and (4.23b) together describe the procedure to obtain the coefficients of $\mathbf{E}^{\mathcal{N}}$.

Now, we are ready to show the asymptotic exactness of the error estimate.

Theorem 4.5. Under the assumptions of Theorem 3.3, let $\mathbf{E}^{\mathcal{R}}$ be computed as specified in Theorem 4.4. Consider the estimate

$$
\mathbf{E}^{\mathcal{N}}(t, h \boldsymbol{\xi})=\sum_{j=1}^{d}\left(L_{p+1}\left(\xi_{j}\right) \boldsymbol{\gamma}_{j}^{\mathcal{N}}(t)-L_{p}\left(\xi_{j}\right) \boldsymbol{\delta}_{j}(t)\right)
$$

where $\boldsymbol{\gamma}_{i}^{\mathcal{N}}, \boldsymbol{\delta}_{i}, 1 \leq i \leq d$, are solutions of (4.25) and 4.23b).

Then, at $t=\mathcal{O}(1)$ and for $p \geq 1$,

$$
\mathbf{e}^{\mathcal{N}}(t, \mathbf{x})=\mathbf{E}^{\mathcal{N}}(t, \mathbf{x})+\mathcal{O}\left(h^{p+2}\right), \quad \mathbf{x} \in \omega .
$$

Proof. Left-multiplying (4.21a) by $\mathbf{R}$ and substituting $\tilde{\mathbf{E}}^{\mathcal{N}}=\mathbf{R E}^{\mathcal{R}}, \tilde{\gamma}_{i}^{\mathcal{N}}=\mathbf{R} \gamma_{i}^{\mathcal{N}}$ and $\tilde{\boldsymbol{\delta}}_{i}=\mathbf{R} \boldsymbol{\delta}_{i}$ yields

$$
\tilde{\mathbf{E}}^{\mathcal{N}}(t, h \boldsymbol{\xi})=\sum_{j=1}^{d}\left(L_{p+1}\left(\xi_{j}\right) \tilde{\boldsymbol{\gamma}}_{j}^{\mathcal{N}}-L_{p}\left(\xi_{j}\right) \tilde{\boldsymbol{\delta}}_{j}\right), \tilde{\boldsymbol{\gamma}}_{i}^{\mathcal{N}}, \tilde{\boldsymbol{\delta}}_{i} \in \mathcal{N}\left(\mathbf{B}_{i}\right), \quad 1 \leq i \leq d,
$$

where we used (3.4a) and the fact that $\boldsymbol{\delta}_{i} \in \mathcal{N}\left(\mathbf{A}_{i}\right)$.

Substituting $\mathbf{w}=\mathbf{R}^{-t} \mathbf{v}, \mathbf{U}_{h}=\mathbf{R} \mathbf{u}_{h}, \tilde{\mathbf{E}}^{\mathcal{N}}=\mathbf{R E}^{\mathcal{N}}, \tilde{\mathbf{E}}^{\mathcal{R}}=\mathbf{R E}^{\mathcal{R}}$ and $\tilde{\mathbf{E}}^{-}=\mathbf{R E}^{-}$ into (4.21b) yields

$$
\begin{aligned}
& \int_{\omega} \mathbf{w}^{t}\left(\frac{\partial\left(\mathbf{U}_{h}+\tilde{\mathbf{E}}^{\mathcal{N}}\right)}{\partial t}+\sum_{j=1}^{d} \mathbf{B}_{j} \frac{\partial \mathbf{U}_{h}}{\partial x_{j}}-\mathbf{R g}\right) d \mathbf{x} \\
& =\sum_{j=1}^{d} \int_{\gamma_{j}} \mathbf{w}^{t} \nu_{j} \mathbf{B}_{j}^{\bar{\mu}_{j}}\left(\mathbf{U}_{h}+\tilde{\mathbf{E}}^{\mathcal{R}}+\tilde{\mathbf{E}}^{\mathcal{N}}-\mathbf{U}_{h}^{-}-\tilde{\mathbf{E}}^{-}\right) d \mathbf{s}, \\
& \forall \mathbf{w}(\mathbf{x})=\sum_{i=1}^{d}\left(L_{p+1}\left(\frac{x_{i}}{h}\right) \mathbf{a}_{i}-L_{p}\left(\frac{x_{i}}{h}\right) \mathbf{b}_{i}\right), \mathbf{a}_{i}, \mathbf{b}_{i} \in \mathcal{N}\left(\mathbf{B}_{i}\right) .
\end{aligned}
$$

where we used (3.4c) and the fact that $\mathbf{v} \in \mathcal{E}_{p}$.

Since system (4.28) satisfies Theorem 4.3 in 8 , we obtain, for $p \geq 1$ and $t=$ $\mathcal{O}(1)$

$$
\tilde{\mathbf{e}}^{\mathcal{N}}(t, \mathbf{x})=\tilde{\mathbf{E}}^{\mathcal{N}}(t, \mathbf{x})+\mathcal{O}\left(h^{p+2}\right), \quad \mathbf{x} \in \omega .
$$


Left-multiplying (4.29) with $\mathbf{R}^{-1}$ and substituting $\tilde{\mathbf{E}}^{\mathcal{N}}=\mathbf{R}^{-1} \mathbf{e}^{\mathcal{N}}$ and $\tilde{\mathbf{E}}^{\mathcal{N}}=$ $\mathbf{R}^{-1} \mathbf{e}^{\mathcal{N}}$ yields (4.27).

\section{Computational examples}

In Section 4 we showed asymptotic exactness of our a posteriori error estimates for a local DG formulation. We will now present computational results for several symmetrizable systems that suggest that our a posteriori error estimates are globally asymptotically exact for smooth solutions.

The accuracy of a posteriori error estimates is measured by the global effectivity index with respect to the $L^{2}$ norm

$$
\theta=\frac{\|\mathbf{E}\|_{2, \Omega}}{\|\mathbf{e}\|_{2, \Omega}}
$$

where $\mathbf{E}$ is either $\mathbf{E}^{\mathcal{R}}$ or $\mathbf{E}^{\mathcal{R}}+\mathbf{E}^{\mathcal{N}}$.

We also need the componentwise $L^{2}$-error,

$$
\|\mathbf{e}\|^{*}=\left(\left\|e_{1}\right\|_{2, \Omega}, \ldots,\left\|e_{m}\right\|_{2, \Omega}\right)^{t},
$$

and the componentwise effectivity index,

$$
\theta^{*}=\left(\frac{\left\|E_{1}\right\|_{2, \Omega}}{\left\|e_{1}\right\|_{2, \Omega}}, \ldots, \frac{\left\|E_{m}\right\|_{2, \Omega}}{\left\|e_{m}\right\|_{2, \Omega}}\right)^{t}
$$

where $\mathbf{E}=\left(E_{1}, \ldots, E_{m}\right)^{t}$ and $\mathbf{e}=\left(e_{1}, \ldots, e_{m}\right)^{t}$.

Ideally, the transient global effectivity indices should approach unity under mesh refinement.

Example 5.1. Let us consider the two-dimensional hyperbolic system

$$
\frac{\partial \mathbf{u}}{\partial t}+\mathbf{A}_{1} \frac{\partial \mathbf{u}}{\partial x_{1}}+\mathbf{A}_{2} \frac{\partial \mathbf{u}}{\partial x_{2}}=\mathbf{g}(t, \mathbf{x}), \mathbf{x} \in(0,1)^{2}, 0<t<1
$$

where

$$
\mathbf{A}_{1}=\left(\begin{array}{ccc}
1 & 1 & -1 \\
1 & 1 & 2 \\
1 & 1 & 2
\end{array}\right), \mathbf{A}_{2}=\left(\begin{array}{ccc}
-6 & 0 & -8 \\
1 & -4 & 7 \\
0 & 0 & 2
\end{array}\right)
$$

and select $\mathbf{g}(t, x, y)$, initial and boundary conditions such that the true solution is

$$
\mathbf{u}(t, \mathbf{x})=\exp \left(t+x_{1}+x_{2}\right)(1,1,1)^{t} .
$$

Note that the system (5.4) is equivalent to the symmetric system for $\mathbf{U}=\mathbf{R u}$

$$
\frac{\partial \mathbf{U}}{\partial t}+\mathbf{B}_{1} \frac{\partial \mathbf{U}}{\partial x_{1}}+\mathbf{B}_{2} \frac{\partial \mathbf{U}}{\partial x_{2}}=\mathbf{R g}(t, \mathbf{x}), \mathbf{x} \in(0,1)^{2}, 0<t<1,
$$

where

$$
\mathbf{B}_{1}=\left(\begin{array}{lll}
2 & 0 & 1 \\
0 & 0 & 0 \\
1 & 0 & 2
\end{array}\right), \mathbf{B}_{2}=\left(\begin{array}{ccc}
-5 & 1 & 0 \\
1 & -5 & 0 \\
0 & 0 & 2
\end{array}\right), \mathbf{R}=\left(\begin{array}{ccc}
1 & 1 & 0 \\
0 & 1 & -1 \\
0 & 0 & 1
\end{array}\right) .
$$

A discussion of the symmetric system (5.5) can be found in 9 . 
Basic linear algebra yields

$$
\begin{aligned}
& \mathcal{R}\left(\mathbf{A}_{1}^{+t}\right) \cap \mathcal{R}\left(\mathbf{A}_{2}^{+t}\right)=\operatorname{span}\left\{(0,0,1)^{t}\right\}, \\
& \mathcal{R}\left(\mathbf{A}_{1}^{+t}\right) \cap \mathcal{R}\left(\mathbf{A}_{2}^{-{ }^{t}}\right)=\operatorname{span}\left\{(1,1,0)^{t}\right\} .
\end{aligned}
$$

Applying Theorem 3.4 we expect $\mathcal{O}\left(h^{p+2}\right)$ pointwise superconvergence of the error projections $e_{3}=(0,0,1)^{t} \mathbf{e}$, and $e_{1}+e_{2}=(1,1,0)^{t} \mathbf{e}$ at Radau points.

We solve (5.4) on uniform meshes having $20^{2}, 40^{2}, 60^{2}, 80^{2}$ elements for $p=1,2,3$. Tables 5.1 and 5.2 present the maximum projected errors for $\left|e_{3}\right|$ at shifted Radau points $\left(\xi_{i}^{+}, \xi_{j}^{+}\right)$and $\left|e_{1}+e_{2}\right|$ at shifted Radau points $\left(\xi_{i}^{+}, \xi_{j}^{-}\right)$at $t=1$, respectively. We observe that the error projections are $\mathcal{O}\left(h^{p+2}\right)$ superconvergent at Radau points, which is in agreement with Theorem 3.4 .

TABle 5.1. Maximum projected errors for $\left|e_{3}\right|$ at shifted Radau points $\left(\xi_{i}^{+}, \xi_{j}^{+}\right)$for Example [5.1 at $t=1$.

\begin{tabular}{|c|ll|ll|ll|}
\hline$N$ & \multicolumn{2}{|c|}{$p=1$} & \multicolumn{2}{c|}{$p=2$} & \multicolumn{2}{c|}{$p=3$} \\
& $\left|e_{3}\right|_{\infty}^{++}$ & order & $\left|e_{3}\right|_{\infty}^{+,+}$ & order & $\left|e_{3}\right|_{\infty}^{++,}$ & order \\
\hline $20^{2}$ & $1.1200 e-3$ & - & $1.8108 e-5$ & - & $5.1874 e-7$ & - \\
$40^{2}$ & $1.4253 e-4$ & 2.9742 & $1.1460 e-6$ & 3.9820 & $1.7717 e-8$ & 4.8718 \\
$60^{2}$ & $4.3063 e-5$ & 2.9518 & $2.2630 e-7$ & 4.0009 & $2.3803 e-9$ & 4.9506 \\
$80^{2}$ & $1.8347 e-5$ & 2.9657 & $7.1624 e-8$ & 3.9989 & $5.6916 e-10$ & 4.9737 \\
\hline
\end{tabular}

TABLE 5.2. Maximum projected errors for $\left|e_{1}+e_{2}\right|$ at shifted Radau points $\left(\xi_{i}^{+}, \xi_{j}^{-}\right)$for Example 5.1 at $t=1$.

\begin{tabular}{|c|ll|ll|ll|}
\hline$N$ & \multicolumn{2}{|c|}{$p=1$} & \multicolumn{2}{c|}{$p=2$} & \multicolumn{2}{c|}{$p=3$} \\
& $\left|e_{1}+e_{2}\right|_{\infty}^{+,-}$ & order & $\left|e_{1}+e_{2}\right|_{\infty}^{+,-}$ & order & $\left|e_{1}+e_{2}\right|_{\infty}^{+,-}$ & order \\
\hline $20^{2}$ & $2.2442 e-3$ & - & $2.2283 e-5$ & - & $1.0707 e-6$ & - \\
$40^{2}$ & $2.9304 e-4$ & 2.9371 & $1.4024 e-6$ & 3.9900 & $3.2800 e-8$ & 5.0287 \\
$60^{2}$ & $8.7966 e-5$ & 2.9678 & $2.8129 e-7$ & 3.9622 & $4.3023 e-9$ & 5.0097 \\
$80^{2}$ & $3.7358 e-5$ & 2.9769 & $8.9331 e-8$ & 3.9872 & $1.0201 e-9$ & 5.0029 \\
\hline
\end{tabular}

We present the componentwise $L^{2}$-errors and effectivity indices corresponding to the stationary error estimate $\mathbf{E}^{\mathcal{R}}$ at $t=1$ in Table 5.3. In Table 5.4, we present the $L^{2}$-errors and global effectivity indices for the transient error estimate $\mathbf{E}^{\mathcal{R}}+\mathbf{E}^{\mathcal{N}}$ at $t=1$. We plot the transient global effectivity indices versus time in Figure 5.1 .

Since $\mathcal{N}\left(\mathbf{A}_{1}\right)=\operatorname{span}\left\{(-1,1,0)^{t}\right\}$ and $\mathcal{N}\left(\mathbf{A}_{2}\right)=\{\mathbf{0}\}$, the stationary error estimate should be asymptotically exact for the third component only. Our numerical results, which show that $\mathbf{E}^{\mathcal{R}}$ is an accurate approximation of the third component of the error, are in full agreement with Theorem 4.4. Furthermore, the transient global effectivity indices stay close to unity at all times, thus the error estimate $\mathbf{E}^{\mathcal{R}}+\mathbf{E}^{\mathcal{N}}$ is asymptotically exact under mesh refinement. Again, our results are in full agreement with Theorem 4.5 . 
TABle 5.3. Componentwise $L^{2}(\Omega)$-errors $\|\mathbf{e}\|^{*},\left\|\mathbf{e}-\mathbf{E}^{\mathcal{R}}\right\|^{*}$ and their order of convergence. Stationary global effectivity indices for Example 5.1 at $t=1$.

\begin{tabular}{|c|c|c|c|c|c|c|}
\hline$p$ & $N$ & $\|\mathbf{e}\|^{*}$ & order & $\left\|\mathbf{e}-\mathbf{E}^{\mathcal{R}}\right\|^{*}$ & order & $\theta^{*}$ \\
\hline \multirow{12}{*}{1} & & $\overline{\Gamma[5.7350 e-3]}$ & & [1.7931e-3] & & 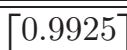 \\
\hline & $20^{2}$ & $3.8792 e-3$ & - & $1.4606 e-3$ & - & 0.7909 \\
\hline & & {$[3.0973 e-3]$} & & {$[4.2058 e-4]$} & & [0.9905] \\
\hline & & $\lceil 1.4135 e-3\rceil$ & $\lceil 2.0205]$ & {$[3.8625 e-4]$} & $\lceil 2.2149]$ & {$[1.0118]$} \\
\hline & $40^{2}$ & $9.6321 e-4$ & 2.0098 & $3.5407 e-4$ & 2.0445 & 0.7951 \\
\hline & & $\lfloor 7.6788 e-4]$ & {$[2.0120\rfloor$} & [5.3478e-5] & [2.9754] & [0.9973] \\
\hline & & $\lceil 6.2607 e-4]$ & {$[2.0085]$} & {$[1.6484 e-4]$} & $\lceil 2.1001\rceil$ & {$[1.0163]$} \\
\hline & $60^{2}$ & $4.2727 e-4$ & 2.0047 & $1.5642 e-4$ & 2.0148 & 0.7964 \\
\hline & & {$[3.4075 e-4]$} & [2.0039] & [1.5917e-5] & [2.9888 & {$[0.9986$} \\
\hline & & {$[3.5166 e-4]$} & {$[2.0050]$} & {$[9.1037 e-5]$} & $\lceil 2.0638]$ & {$[1.0181]$} \\
\hline & $80^{2}$ & $2.4013 e-4$ & 2.0031 & $8.7774 e-5$ & 2.0085 & 0.7971 \\
\hline & & [1.9157e-4] & [2.0019] & [6.7335e-6] & {$[2.9905$} & 0.9992 \\
\hline \multirow{12}{*}{2} & & {$[9.4899 e-5]$} & & {$[2.9179 e-5]$} & & 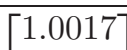 \\
\hline & $20^{2}$ & $6.4873 e-5$ & - & $2.3785 e-5$ & - & 0.8066 \\
\hline & & {$[5.2935 e-5]$} & & $8.9282 e-6$ & & [0.9886 \\
\hline & & {$[1.1742 e-5]$} & {$[3.0147]$} & {$[3.0753 e-6]$} & $\lceil 3.2462]$ & {$[1.0168]$} \\
\hline & $40^{2}$ & $8.0523 e-6$ & 3.0102 & $2.7999 e-6$ & 3.0866 & 0.8134 \\
\hline & & [6.5681e-6] & [3.0107] & [5.6091e-7] & [3.9925 & [0.9972 \\
\hline & & {$[3.4710 e-6]$} & {$[3.0058]$} & {$[8.6725 e-7]$} & {$[3.1219]$} & {$[1.0201]$} \\
\hline & $60^{2}$ & $2.3817 e-6$ & 3.0043 & $8.1833 e-7$ & 3.0338 & 0.8149 \\
\hline & & {$[1.9433 e-6]$} & [3.0036] & [1.1096e-7] & [3.9963] & [0.9988 \\
\hline & & {$[1.4629 e-6]$} & {$[3.0034]$} & {$[3.5788 e-7]$} & {$[3.0768]$} & {$[1.0214]$} \\
\hline & $80^{2}$ & $1.0041 e-6$ & 3.0026 & $3.4335 e-7$ & 3.0190 & 0.8155 \\
\hline & & [8.1940e-7] & [3.0018] & [3.5134e-8] & 3.9976 & [0.9993 \\
\hline \multirow{12}{*}{3} & & {$[1.3609 e-6]$} & & {$[4.9842 e-7]$} & & {$[0.9862]$} \\
\hline & $20^{2}$ & $9.3751 e-7$ & - & $3.8953 e-7$ & - & 0.7809 \\
\hline & & {$[7.6992 e-7]$} & & {$[2.1759 e-7]$} & & {$[0.9509]$} \\
\hline & & {$[8.3042 e-8]$} & {$[4.0346]$} & {$[2.3848 e-8]$} & $\lceil 4.3855]$ & {$[1.0108]$} \\
\hline & $40^{2}$ & $5.7629 e-8$ & 4.0240 & $2.1379 e-8$ & 4.1875 & 0.8034 \\
\hline & & [4.6996e-8] & [4.0341] & [7.1458e-9] & [4.9284] & [0.9852] \\
\hline & & {$[1.6322 e-8]$} & $\lceil 4.0123]$ & {$[4.3560 e-9]$} & {$[4.1930]$} & {$[1.0159]$} \\
\hline & $60^{2}$ & $1.1342 e-8$ & 4.0089 & $4.0937 e-9$ & 4.0767 & 0.8087 \\
\hline & & {$[9.2372 e-9]$} & [4.0122] & {$[9.5490 e-10$} & [4.9639] & [0.9930] \\
\hline & & {$[5.1545 e-9]$} & $\lceil 4.0066\rceil$ & {$[1.3340 e-9$} & {$[4.1134]$} & {$[1.0179]$} \\
\hline & $80^{2}$ & $3.5838 e-9$ & 4.0048 & $1.2804 e-9$ & 4.0403 & 0.8108 \\
\hline & & $2.9175 e-9$ & 4.0063 & $2.2807 e-10$ & 4.9776 & 0.9959 \\
\hline
\end{tabular}


TABLe 5.4. $L^{2}$-errors $\|\mathbf{e}\|_{2, \Omega},\left\|\mathbf{e}-\mathbf{E}^{\mathcal{R}}-\mathbf{E}^{\mathcal{N}}\right\|_{2, \Omega}$ and their order of convergence. Transient global effectivity indices for Example 5.1 at $t=1$.

\begin{tabular}{|c|c|cc|cc|c|}
\hline$p$ & $N$ & $\|\mathbf{e}\|$ & order & $\left\|\mathbf{e}-\mathbf{E}^{\mathcal{R}}-\mathbf{E}^{\mathcal{N}}\right\|$ & order & $\theta$ \\
\hline \hline & $20^{2}$ & $7.5850 e-3$ & - & $1.0329 e-3$ & - & 0.9828 \\
& $40^{2}$ & $1.8749 e-3$ & 2.016 & $1.3030 e-4$ & 2.987 & 0.9947 \\
& $60^{2}$ & $8.3105 e-4$ & 2.007 & $3.8708 e-5$ & 2.994 & 0.9974 \\
& $80^{2}$ & $4.6693 e-4$ & 2.004 & $1.6346 e-5$ & 2.997 & 0.9985 \\
\hline \hline \multirow{4}{*}{2} & $20^{2}$ & $1.2656 e-4$ & - & $2.0218 e-5$ & - & 0.9824 \\
& $40^{2}$ & $1.5680 e-5$ & 3.013 & $1.2696 e-6$ & 3.993 & 0.9944 \\
& $60^{2}$ & $4.6365 e-6$ & 3.005 & $2.5101 e-7$ & 3.998 & 0.997 \\
& $80^{2}$ & $1.9544 e-6$ & 3.003 & $7.9435 e-8$ & 3.999 & 0.9981 \\
\hline \hline \multirow{4}{*}{3} & $20^{2}$ & $1.8232 e-6$ & - & $4.6946 e-7$ & - & 0.9618 \\
& $40^{2}$ & $1.1147 e-7$ & 4.032 & $1.5159 e-8$ & 4.953 & 0.9886 \\
& $60^{2}$ & $2.1917 e-8$ & 4.011 & $2.0158 e-9$ & 4.976 & 0.9944 \\
& $80^{2}$ & $6.9227 e-9$ & 4.006 & $4.8042 e-10$ & 4.985 & 0.9966 \\
\hline
\end{tabular}
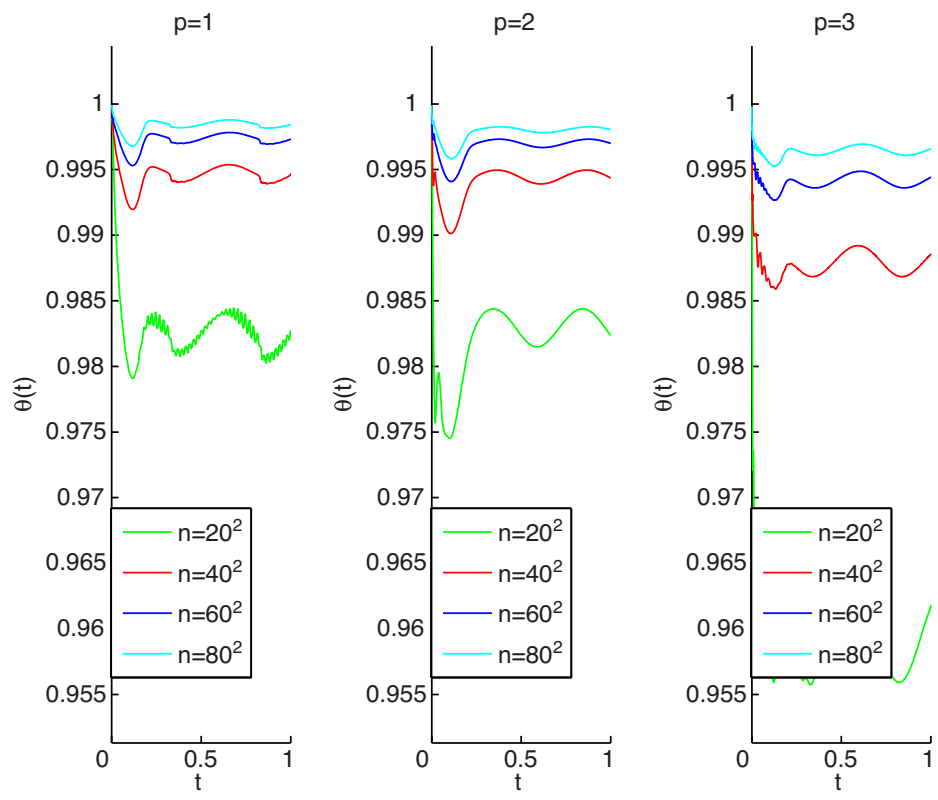

Figure 5.1. Transient global effectivity indices versus time for Example 5.1. 
Example 5.2. Let us consider Maxwell's equations of electromagnetism,

$$
\begin{aligned}
\varepsilon_{0} \frac{\partial \mathcal{E}}{\partial t} & =\nabla \times \mathcal{H}, \quad \mu_{0} \frac{\partial \mathcal{H}}{\partial t}=\nabla \times \mathcal{E}, \\
\nabla \cdot \mathcal{E} & =0, \quad \nabla \cdot \mathcal{H}=0,
\end{aligned}
$$

where $\mathcal{E}(t, \mathbf{x})=\left(\mathcal{E}_{1}, \mathcal{E}_{2}, \mathcal{E}_{3}\right)^{t}$ and $\mathcal{H}(t, \mathbf{x})=\left(\mathcal{H}_{1}, \mathcal{H}_{2}, \mathcal{H}_{3}\right)^{t}$ denote the electric and magnetic field and $\mu_{0}=4 \pi \cdot 10^{-7} N A^{-2}$ and $\varepsilon_{0}=c_{0}^{-2} \mu_{0}^{-1}$ denote the magnetic and electric permittivity in free space, respectively, with $c_{0}=299,792,458 \mathrm{~ms}^{-2}$ being the speed of light. Equations (5.7b) are satisfied for all $t$, if the initial conditions satisfy them.

For a transverse electric wave traveling in the $x_{1} x_{2}$-plane, $\mathcal{E}_{3}=\mathcal{H}_{1}=\mathcal{H}_{2}=0$, and equation (5.7a) yields the linear symmetrizable hyperbolic system

$$
\frac{\partial \mathbf{u}}{\partial t}+\mathbf{A}_{1} \frac{\partial \mathbf{u}}{\partial x_{1}}+\mathbf{A}_{2} \frac{\partial \mathbf{u}}{\partial x_{2}}=\mathbf{g}, \mathbf{x} \in \Omega=(0,3)^{2}, 0<t<6 \cdot 10^{-7} s,
$$

where

$$
\mathbf{u}=\left(\begin{array}{c}
\mathcal{E}_{x} \\
\mathcal{E}_{y} \\
\mathcal{H}_{z}
\end{array}\right), \quad \mathbf{A}_{1}=\left(\begin{array}{ccc}
0 & 0 & 0 \\
0 & 0 & \varepsilon_{0}^{-1} \\
0 & \mu_{0}^{-1} & 0
\end{array}\right), \quad \mathbf{A}_{2}=\left(\begin{array}{ccc}
0 & 0 & -\varepsilon_{0}^{-1} \\
0 & 0 & 0 \\
-\mu_{0}^{-1} & 0 & 0
\end{array}\right)
$$

We select initial and boundary conditions such that the true solution is a typical FM radio wave of wavelength $\lambda=3 m$ and amplitude $A=1.5 \mathrm{~V} / \mathrm{m}$ traveling in the direction $\left(x_{1}, x_{2}\right)=(1,1)$,

$$
\mathbf{u}(t, \mathbf{x})=\left(1,-1, \sqrt{2 \mu_{0} \varepsilon_{0}^{-1}}\right)^{t} A \sin \left(\frac{2 \pi}{\lambda}\left(c_{0} t+\frac{x+y}{\sqrt{2}}\right)\right)
$$

Applying Theorem 4.4, the stationary error estimate $\mathbf{E}^{\mathcal{R}}$ can only accurately approximate the component of the error lying in $\left(\mathcal{N}\left(\mathbf{A}_{1}\right) \bigoplus \mathcal{N}\left(\mathbf{A}_{2}\right)\right)^{\perp}=$ $\operatorname{span}\left\{(0,0,1)^{t}\right\}$, i.e., only $E_{3}^{\mathcal{R}}$ is an accurate estimate of $e_{3}$.

To validate our theory for short and long time integration, we solve (5.8) on uniform meshes having $N=10^{2}, 20^{2}, 40^{2}$ elements for $p=1,2,3$ on $0 \leq t \leq 3 \cdot 10^{-8} \mathrm{~s}$ and $0 \leq t \leq 6 \cdot 10^{-7} \mathrm{~s}$, respectively, where the wave travels through the domain three and 60 times. We present the componentwise $L^{2}$-errors and effectivity indices corresponding to the stationary error estimate $\mathbf{E}^{\mathcal{R}}$ at $t=6 \cdot 10^{-7}$ in Table 5.5. In Table 5.6 we present $L^{2}$-errors and global effectivity indices for the transient error estimate $\mathbf{E}^{\mathcal{R}}+\mathbf{E}^{\mathcal{N}}$ at $t=6 \cdot 10^{-7}$. We plot the transient global effectivity indices versus time for $0 \leq t \leq 3 \cdot 10^{-8}$ in Figure 5.2 and for $0 \leq t \leq 6 \cdot 10^{-7}$ in Figure 5.3. In Figure 5.4, we plot the $L^{2}$-errors $\|\mathbf{e}\|_{2, \Omega}$ and $\left\|\mathbf{E}^{\mathcal{R}}+\mathbf{E}^{\mathcal{\mathcal { N }}}\right\|_{2, \Omega}$ versus time for $N=40$.

We observe that $\mathbf{E}^{\mathcal{R}}$ is an accurate approximation of the error only in the third component, which is in agreement with Theorem 4.4. Furthermore, the transient global effectivity indices converge to unity under mesh refinement and stay close to unity at all times, which is in agreement with Theorem 4.5. In Figures 5.25 .4 , 
we observe an oscillation of the $L^{2}$-error and the transient global effectivity index that decays in time. We further observe that the transient global effectivity index converges to unity under mesh refinement and with increasing time for both short and long times.

TABLE 5.5. Componentwise $L^{2}(\Omega)$-errors $\|\mathbf{e}\|^{*},\left\|\mathbf{e}-\mathbf{E}^{\mathcal{R}}\right\|^{*}$ and their order of convergence. Stationary global effectivity indices for Example 5.2 at $t=6 \cdot 10^{-7}$.

\begin{tabular}{|c|c|c|c|c|c|c|}
\hline$p$ & $N$ & $\|\mathbf{e}\|^{*}$ & order & $\left\|\mathbf{e}-\mathbf{E}^{\mathcal{R}}\right\|^{*}$ & order & $\theta^{*}$ \\
\hline \multirow{9}{*}{1} & & 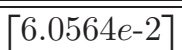 & & [3.6643e-2] & & {$[0.8110]$} \\
\hline & $10^{2}$ & $6.0564 e-2$ & - & $3.6643 e-2$ & - & 0.8110 \\
\hline & & {$[1.7895 e-4]$} & & {$[7.1024 e-5$} & & {$[0.9559$} \\
\hline & & {$[1.4784 e-2]$} & {$[2.0344]$} & {$[8.2752 e-3]$} & {$[2.1467]$} & {$[0.8327]$} \\
\hline & $20^{2}$ & $1.4784 e-2$ & 2.0344 & $8.2752 e-3$ & 2.1467 & 0.8327 \\
\hline & & {$[4.3088 e-5]$} & [2.0541] & {$[8.8014 e-6]$} & [3.0125] & {$[0.9887]$} \\
\hline & & {$[3.6645 e-3]$} & {$[2.0124]$} & {$[1.9939 e-3]$} & {$[2.0532]$} & {$[0.8405]$} \\
\hline & $40^{2}$ & $3.6645 e-3$ & 2.0124 & $1.9939 e-3$ & 2.0532 & 0.8405 \\
\hline & & {$[1.0666 e-5]$} & [2.0143] & {$[1.0888 e-6]$} & [3.0150 & {$[0.9977$} \\
\hline \multirow{9}{*}{2} & & {$[2.0624 e-3]$} & & {$[1.2042 e-3]$} & & $\lceil 0.8136]$ \\
\hline & $10^{2}$ & $2.0624 e-3$ & - & $1.2042 e-3$ & - & 0.8136 \\
\hline & & {$[6.0813 e-6]$} & & [1.5271e-6] & & {$[0.9815$} \\
\hline & & {$[2.5458 e-4]$} & {$[3.0181]$} & {$[1.4211 e-4]$} & {$[3.0830]$} & {$[0.8294]$} \\
\hline & $20^{2}$ & $2.5458 e-4$ & 3.0181 & $1.4211 e-4$ & 3.0830 & 0.8294 \\
\hline & & {$[7.5420 e-7]$} & 3.0114 & {$[9.6012 e-8]$} & [3.9915] & {$[0.9945$} \\
\hline & & {$[3.1538 e-5]$} & {$[3.0130]$} & $\lceil 1.7167 e-5]$ & {$[3.0492]$} & {$[0.8384]$} \\
\hline & $40^{2}$ & $3.1538 e-5$ & 3.0130 & $1.7167 e-5$ & 3.0492 & 0.8384 \\
\hline & & {$[9.4076 e-8]$} & {$[3.0031]$} & {$[6.0238 e-9$} & [3.9945] & {$[0.9981$} \\
\hline \multirow{9}{*}{3} & & {$[5.8930 e-5]$} & & {$[3.6614 e-5]$} & & {$[0.7933]$} \\
\hline & $10^{2}$ & $5.8930 e-5$ & - & $3.6614 e-5$ & - & 0.7933 \\
\hline & & {$[1.7386 e-7]$} & & [5.2173e-8] & & {$[0.9699]$} \\
\hline & & {$[3.5663 e-6]$} & {$[4.0465]$} & {$[2.0417 e-6]$} & $\lceil 4.1646]$ & {$[0.8230]$} \\
\hline & $20^{2}$ & $3.5663 e-6$ & 4.0465 & $2.0417 e-6$ & 4.1646 & 0.8230 \\
\hline & & {$[1.0649 e-8]$} & [4.0291] & [1.6389e-9] & [4.9925] & [0.9929 \\
\hline & & {$[2.2006 e-7]$} & {$[4.0185]$} & {$[1.2152 e-7]$} & {$[4.0706]$} & {$[0.8345]$} \\
\hline & $40^{2}$ & $2.2006 e-7$ & 4.0185 & $1.2152 e-7$ & 4.0706 & 0.8345 \\
\hline & & {$[6.6243 e-10]$} & 4.0068 & {$[5.1297 e-11]$} & [4.9977] & {$[0.9983$} \\
\hline
\end{tabular}


TABLE 5.6. $L^{2}$-errors $\|\mathbf{e}\|_{2, \Omega},\left\|\mathbf{e}-\mathbf{E}^{\mathcal{R}}-\mathbf{E}^{\mathcal{N}}\right\|_{2, \Omega}$ and their order of convergence. Transient global effectivity indices for Example 5.2 at $t=6 \cdot 10^{-7}$.

\begin{tabular}{|c|c|cc|cc|c|}
\hline$p$ & $N$ & $\|\mathbf{e}\|$ & order & $\left\|\mathbf{e}-\mathbf{E}^{\mathcal{R}}-\mathbf{E}^{\mathcal{N}}\right\|$ & order & $\theta$ \\
\hline \hline & $10^{2}$ & $8.5650 e-2$ & - & $3.0040 e-2$ & - & 0.9509 \\
1 & $20^{2}$ & $2.0908 e-2$ & 2.034 & $3.9764 e-3$ & 2.917 & 0.9891 \\
& $40^{2}$ & $5.1823 e-3$ & 2.012 & $6.2109 e-4$ & 2.679 & 1.009 \\
\hline \hline \multirow{3}{*}{2} & $10^{2}$ & $2.9166 e-3$ & - & $8.8179 e-4$ & - & 0.9531 \\
& $20^{2}$ & $3.6004 e-4$ & 3.018 & $6.0954 e-5$ & 3.855 & 0.9873 \\
& $40^{2}$ & $4.4602 e-5$ & 3.013 & $5.2913 e-6$ & 3.526 & 1.007 \\
\hline \hline \multirow{3}{*}{3} & $10^{2}$ & $8.3340 e-5$ & - & $3.1830 e-5$ & - & 0.9346 \\
& $20^{2}$ & $5.0436 e-6$ & 4.046 & $1.0590 e-6$ & 4.91 & 0.9855 \\
& $40^{2}$ & $3.1122 e-7$ & 4.018 & $4.2565 e-8$ & 4.637 & 1.006 \\
\hline
\end{tabular}
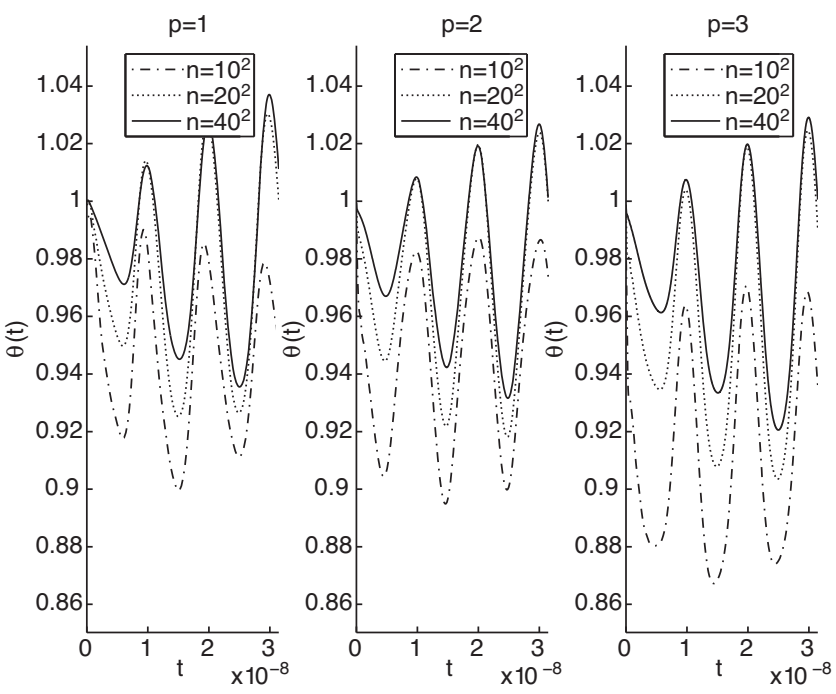

Figure 5.2. Transient global effectivity indices versus time for Example 5.2.

Example 5.3. Let us consider the equation of an acoustic wave in dry air at $20^{\circ} \mathrm{C}$,

$$
\frac{\partial \rho}{\partial t}+\nabla \cdot\left(\rho_{0} \mathbf{v}\right)=0, \frac{\partial \mathbf{v}}{\partial t}+\nabla\left(\frac{c^{2}}{\rho_{0}} \rho\right)=\mathbf{0}
$$

where $\rho$ is the density, $\mathbf{v}$ the velocity field, $\rho_{0}=1.2 \mathrm{~kg} / \mathrm{m}^{3}$ the reference density, and $c_{0}=340 \mathrm{~m} / \mathrm{s}$ the speed of sound. In two dimensions, system (5.9) can be written in symmetrizable hyperbolic form as

$$
\frac{\partial \mathbf{u}}{\partial t}+\mathbf{A}_{1} \frac{\partial \mathbf{u}}{\partial x_{1}}+\mathbf{A}_{2} \frac{\partial \mathbf{u}}{\partial x_{2}}=\mathbf{0}, \mathbf{x} \in \Omega=(0,2 \sqrt{2} \pi)^{2}, 0<t<1,
$$



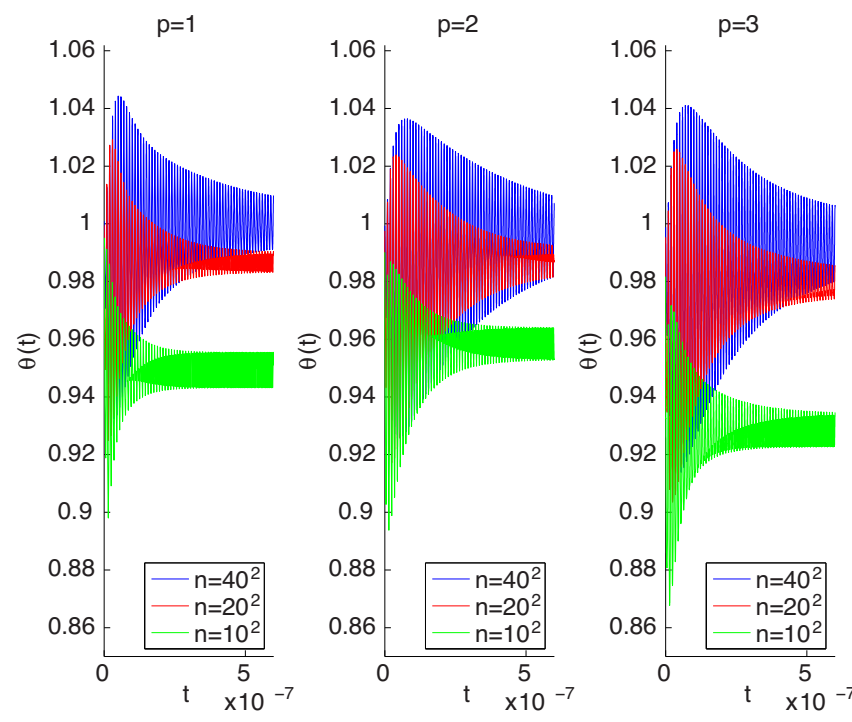

Figure 5.3. Transient global effectivity indices versus time for Example 5.2.
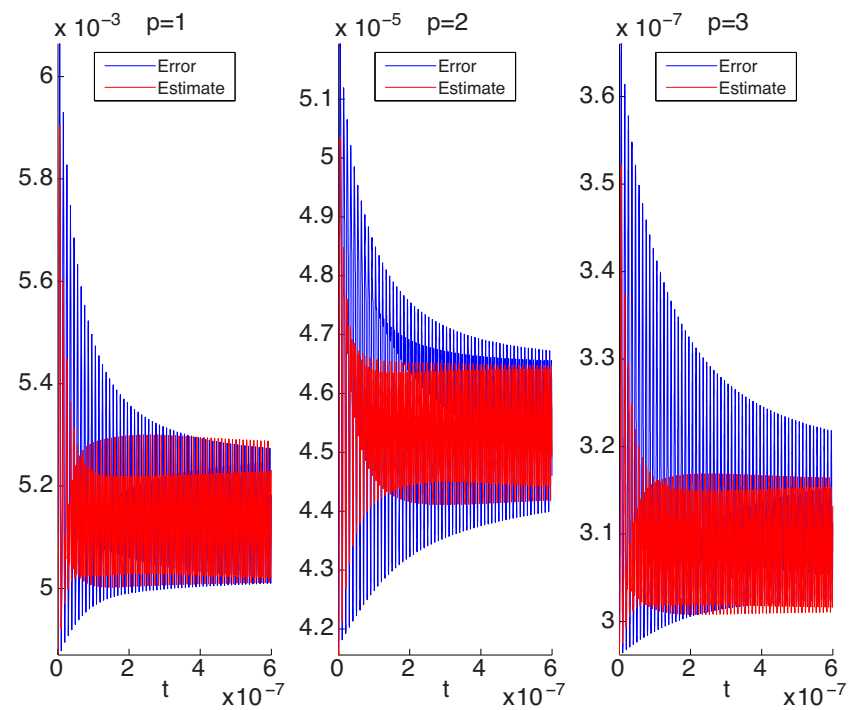

Figure 5.4. $L^{2}$-errors $\|\mathbf{e}\|_{2, \Omega}$ and $\left\|\mathbf{E}^{\mathcal{R}}+\mathbf{E}^{\mathcal{N}}\right\|_{2, \Omega}$ versus time for Example 5.2 with $N=40$.

where

$$
\mathbf{u}=\left(\begin{array}{c}
\rho \\
v_{1} \\
v_{2}
\end{array}\right), \mathbf{A}_{1}=\left(\begin{array}{ccc}
0 & \rho_{0} & 0 \\
\frac{c_{0}^{2}}{\rho_{0}} & 0 & 0 \\
0 & 0 & 0
\end{array}\right), \mathbf{A}_{2}=\left(\begin{array}{ccc}
0 & 0 & \rho_{0} \\
0 & 0 & 0 \\
\frac{c_{0}^{2}}{\rho_{0}} & 0 & 0
\end{array}\right)
$$


We select initial and boundary conditions such that the true solution is

$$
\begin{aligned}
& \rho(t, \mathbf{x})=\sin \left(c_{0} t-\frac{x_{1}+x_{2}}{\sqrt{2}}\right), \\
& \mathbf{v}(t, \mathbf{x})=\frac{c_{0}}{\sqrt{2} \rho_{0}} \sin \left(c_{0} t-\frac{x_{1}+x_{2}}{\sqrt{2}}\right)(1,1)^{t} .
\end{aligned}
$$
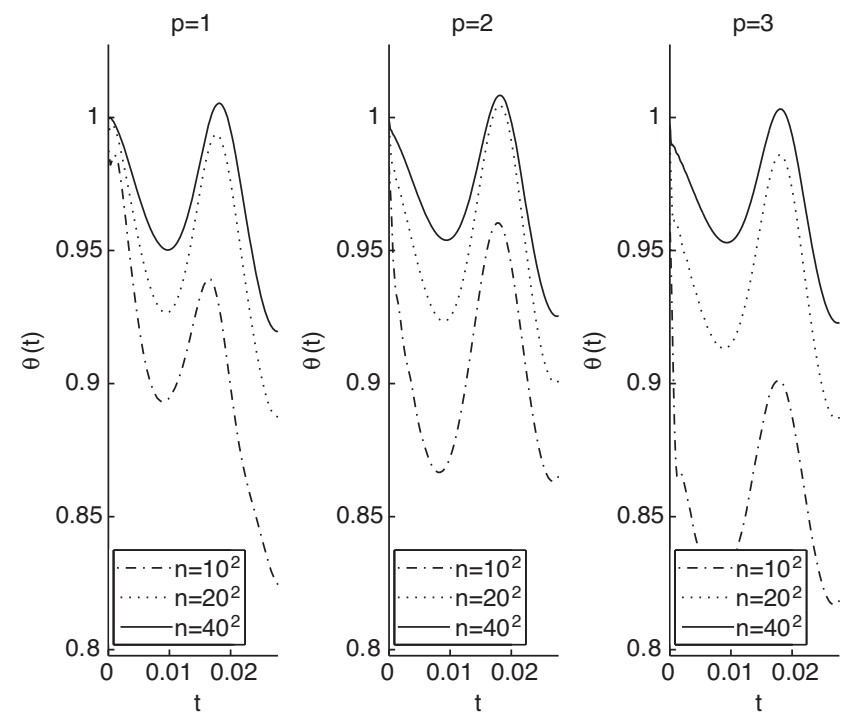

FiguRe 5.5. Transient global effectivity indices versus time for $0 \leq t \leq \frac{3 \pi}{340}$ for Example 5.3

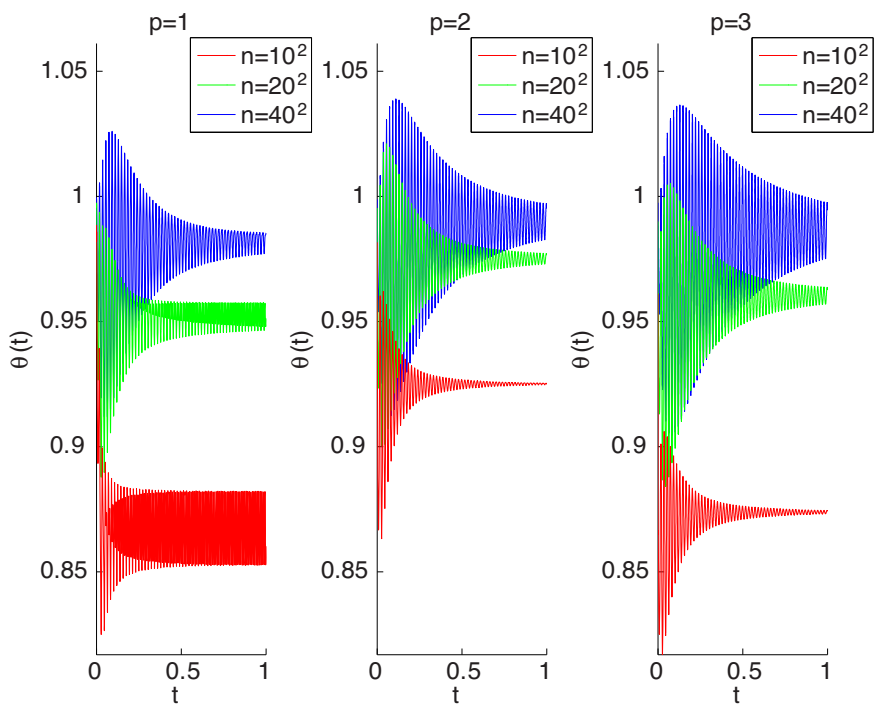

FiguRE 5.6. Transient global effectivity indices versus time for $0 \leq t \leq 1$ for Example 5.3 . 

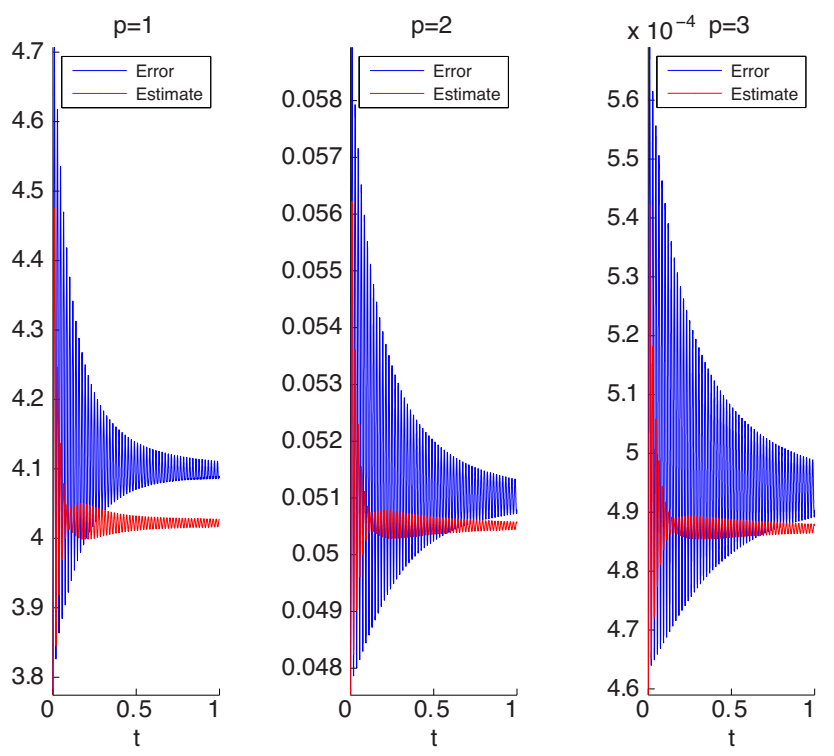

Figure 5.7. $L^{2}$-errors $\|\mathbf{e}\|_{2, \Omega}$ and $\left\|\mathbf{E}^{\mathcal{R}}+\mathbf{E}^{\mathcal{N}}\right\|_{2, \Omega}$ versus time for $n=40^{2}$ and $0 \leq t \leq 1$ for Example 5.3 .

Applying Theorem 4.4, the stationary error estimate $\mathbf{E}^{\mathcal{R}}$ can only accurately approximate the component of the error lying in $\left(\bigoplus_{i=1}^{3} \mathcal{N}\left(\mathbf{A}_{i}\right)\right)^{\perp}=\operatorname{span}\left\{(1,0,0)^{t}\right\}$, i.e., only $E_{1}^{\mathcal{R}}$ is an accurate estimate of $e_{1}$.

We solve (5.10) on uniform meshes having $N=10^{2}, 20^{2}, 40^{2}$ elements for $p=$ $1,2,3$ on $0 \leq t \leq 1$ where the wave travels through the domain 38 times. We present the componentwise $L^{2}$-errors and effectivity indices corresponding to the stationary error estimate $\mathbf{E}^{\mathcal{R}}$ at $t=1$ in Table 5.7. In Table 5.8, we present the $L^{2}$-errors and global effectivity indices for the transient error estimate $\mathbf{E}^{\mathcal{R}}+\mathbf{E}^{\mathcal{N}}$ at $t=1$. We plot the transient global effectivity indices versus time on a short time interval in Figure 5.5 and for a long time interval in Figure 5.6. In Figure 5.7 we plot the $L^{2}$-errors $\|\mathbf{e}\|_{2, \Omega}$ and $\left\|\mathbf{E}^{\mathcal{R}}+\mathbf{E}^{\mathcal{N}}\right\|_{2, \Omega}$.

We observe that $\mathbf{E}^{\mathcal{R}}$ is an accurate approximation of the third component of true error, which is in agreement with Theorem 4.4 while the transient error estimate $\mathbf{E}^{\mathcal{R}}+\mathbf{E}^{\mathcal{N}}$ accurately approximates the error in all components with transient global effectivity indices converging to unity under mesh refinement. This is in agreement with Theorem 4.5. In Figures 5.55 5.7, we observe an oscillation of the $L^{2}$-error and the transient global effectivity index that decays in time. We observe that the transient global effectivity index converges to unity under both mesh refinement and increasing time. 
TABle 5.7. Componentwise $L^{2}(\Omega)$-errors $\|\mathbf{e}\|^{*},\left\|\mathbf{e}-\mathbf{E}^{\mathcal{R}}\right\|^{*}$ and their order of convergence. Stationary global effectivity indices for Example 5.3 at $t=1$.

\begin{tabular}{|c|c|c|c|c|c|c|}
\hline$p$ & $N$ & $\|\mathbf{e}\|^{*}$ & order & $\left\|\mathbf{e}-\mathbf{E}^{\mathcal{R}}\right\|^{*}$ & order & $\theta^{*}$ \\
\hline \multirow{9}{*}{1} & & {$[2.0690 e-1$} & & {$[1.2549 e-1]$} & & {$[0.8632$} \\
\hline & $10^{2}$ & 51.236 & - & 35.889 & - & 0.7463 \\
\hline & & 51.236 & & 35.889 & & 0.7463 \\
\hline & & $=4.6514 e-2$ & {$[2.1532]$} & {$[1.6000 e-2]$} & {$[2.9714]$} & {$[0.9530]$} \\
\hline & $20^{2}$ & 11.860 & 2.1111 & 7.0454 & 2.3488 & 0.8108 \\
\hline & & 11.860 & 2.1111 & 7.0454 & 2.3488 & 0.8108 \\
\hline & & {$[1.1218 e-2$} & {$[2.0519]$} & {$[2.0224 e-3]$} & {$[2.9840$} & {$\left[0.9865^{\top}\right.$} \\
\hline & $40^{2}$ & 2.8901 & 2.0369 & 1.6039 & 2.1351 & 0.8332 \\
\hline & & 2.8901 & 2.0369 & 1.6039 & 2.1351 & 0.8332 \\
\hline \multirow{9}{*}{2} & & {$[9.2211 e-3$} & & {$[3.1815 e-3]$} & & {$[0.9715$} \\
\hline & $10^{2}$ & 2.3640 & - & 1.4531 & - & 0.7988 \\
\hline & & 2.3640 & & 1.4531 & & 0.7988 \\
\hline & & $=1.1363 e-3$ & {$[3.0206]$} & {$[1.9998 e-4]$} & {$[3.9918$} & 0.9930 \\
\hline & $20^{2}$ & $2.8941 e-1$ & 3.0300 & $1.6465 e-1$ & 3.1416 & 0.8248 \\
\hline & & $2.8941 e-1$ & 3.0300 & $1.6465 e-1$ & 3.1416 & 0.8248 \\
\hline & & $1.4156 e-4$ & 3.0049 & {$[1.2523 e-5]$} & 3.9972 & 0.9982 \\
\hline & $40^{2}$ & $3.5919 e-2$ & 3.0103 & $1.9897 e-2$ & 3.0488 & 0.8331 \\
\hline & & $3.5919 e-2$ & 3.0103 & {$[1.9897 e-2]$} & 3.0488 & 0.8331 \\
\hline \multirow{9}{*}{3} & & $3.7275 e-4$ & & $1.5589 e-4$ & & 0.9330 \\
\hline & $10^{2}$ & $9.6281 e-2$ & - & $6.4869 e-2$ & - & 0.7525 \\
\hline & & $9.6281 e-2$ & & $6.4869 e-2$ & & 0.7525 \\
\hline & & {$[2.2347 e-5$} & {$[4.0601]$} & {$[4.9216 e-6]$} & {$[4.9852]$} & $0.9817^{7}$ \\
\hline & $20^{2}$ & $5.6608 e-3$ & 4.0882 & $3.3523 e-3$ & 4.2743 & 0.8090 \\
\hline & & $5.6608 e-3$ & 4.0882 & $3.3523 e-3]$ & 4.2743 & 0.8090 \\
\hline & & {$[1.3809 e-6$} & {$[4.0164]$} & {$[1.5429 e-7]$} & 4.9954 & {$[0.9953$} \\
\hline & $40^{2}$ & $3.4671 e-4$ & 4.0292 & $1.9481 e-4$ & 4.1050 & 0.8279 \\
\hline & & $3.4671 e-4$ & 4.0292 & $1.9481 e-4$ & 4.1050 & 0.8279 \\
\hline
\end{tabular}

TABLe 5.8. $L^{2}$-errors $\|\mathbf{e}\|_{2, \Omega},\left\|\mathbf{e}-\mathbf{E}^{\mathcal{R}}-\mathbf{E}^{\mathcal{N}}\right\|_{2, \Omega}$ and their order of convergence. Transient global effectivity indices for Example 5.3 at $t=1$.

\begin{tabular}{|c|c|cc|cc|c|}
\hline$p$ & $N$ & $\|\mathbf{e}\|$ & order & $\left\|\mathbf{e}-\mathbf{E}^{\mathcal{R}}-\mathbf{E}^{\mathcal{N}}\right\|$ & order & $\theta$ \\
\hline \hline \multirow{3}{*}{1} & $10^{2}$ & 72.459 & - & 40.492 & - & 0.8603 \\
& $20^{2}$ & 16.772 & 2.111 & 5.1955 & 2.962 & 0.9511 \\
& $40^{2}$ & 4.0873 & 2.037 & $6.8190 e-1$ & 2.93 & 0.9854 \\
\hline \hline \multirow{3}{*}{2} & $10^{2}$ & 3.3433 & - & 1.3193 & - & 0.9249 \\
& $20^{2}$ & $4.0930 e-1$ & 3.03 & $8.8297 e-2$ & 3.901 & 0.9765 \\
& $40^{2}$ & $5.0798 e-2$ & 3.01 & $6.3763 e-3$ & 3.792 & 0.9952 \\
\hline \hline \multirow{3}{*}{3} & $10^{2}$ & $1.3616 e-1$ & - & $6.9317 e-2$ & - & 0.8747 \\
& $20^{2}$ & $8.0057 e-3$ & 4.088 & $2.2749 e-3$ & 4.929 & 0.9629 \\
& $40^{2}$ & $4.9032 e-4$ & 4.029 & $7.8954 e-5$ & 4.849 & 0.9947 \\
\hline
\end{tabular}


Example 5.4. In three dimensions, (5.9) can be written as the symmetrizable hyperbolic system

$$
\mathbf{u}_{, t}+\sum_{i=1}^{3} \mathbf{A}_{i} \frac{\partial \mathbf{u}}{\partial x_{i}}=\mathbf{0}, \mathbf{x} \in \Omega=(0,1)^{3}, 0<t<10^{-2},
$$

where $\mathbf{u}=\left(\rho, v_{1}, v_{2}, v_{3}\right)^{t}$ and

$$
\mathbf{A}_{1}=\left(\begin{array}{cccc}
0 & \rho_{0} & 0 & 0 \\
\frac{c_{0}^{2}}{\rho_{0}} & 0 & 0 & 0 \\
0 & 0 & 0 & 0 \\
0 & 0 & 0 & 0
\end{array}\right), \mathbf{A}_{2}=\left(\begin{array}{cccc}
0 & 0 & \rho_{0} & 0 \\
0 & 0 & 0 & 0 \\
\frac{c^{2}}{\rho_{0}} & 0 & 0 & 0 \\
0 & 0 & 0 & 0
\end{array}\right), \mathbf{A}_{3}=\left(\begin{array}{cccc}
0 & 0 & 0 & \rho_{0} \\
0 & 0 & 0 & 0 \\
0 & 0 & 0 & 0 \\
\frac{c^{2}}{\rho_{0}} & 0 & 0 & 0
\end{array}\right)
$$

We select initial and boundary conditions such that the true solution is

$$
\begin{aligned}
\rho(t, \mathbf{x}) & =\exp \left(c_{0} t-\left(x_{1}+x_{2}+x_{3}\right) / \sqrt{3}\right), \\
\mathbf{v}(t, \mathbf{x}) & =\frac{c_{0}}{\sqrt{3} \rho_{0}} \exp \left(c_{0} t-\left(x_{1}+x_{2}+x_{3}\right) / \sqrt{3}\right)(1,1,1)^{t} .
\end{aligned}
$$

Basic linear algebra shows that $\left(\bigoplus_{i=1}^{3} \mathcal{N}\left(\mathbf{A}_{i}\right)\right)^{\perp}=\operatorname{span}\left\{(1,0,0,0)^{t}\right\}$. Applying our theory, the stationary error estimate $\mathbf{E}^{\mathcal{R}}$ can only accurately approximate the first component of the error, i.e., only $E_{1}^{\mathcal{R}}$ is an accurate estimate of $e_{1}$.

We solve (5.11) on uniform meshes having $N=10^{3}, 15^{3}, 20^{3}$ elements for $p=$ $1,2,3$ on $0 \leq t \leq 10^{-2}$. We present the componentwise $L^{2}$-errors and effectivity indices corresponding to the stationary error estimate $\mathbf{E}^{\mathcal{R}}$ at $t=10^{-2}$ in Tables 5.9 and 5.10. In Tables 5.11 and 5.12, we present the componentwise and global $L^{2}$ errors and effectivity indices for the transient error estimate $\mathbf{E}^{\mathcal{R}}+\mathbf{E}^{\mathcal{N}}$ at $t=10^{-2}$. We show the behavior of the error estimate versus time by plotting the transient global effectivity indices versus time in Figure 5.8
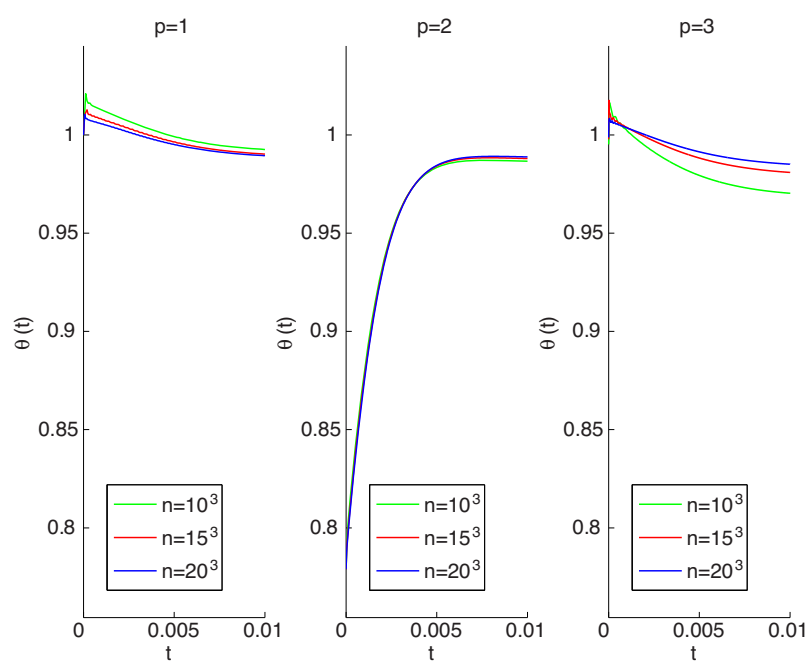

Figure 5.8. Transient global effectivity indices versus time for Example 5.4. 
We observe that $\mathbf{E}^{\mathcal{R}}$ is an accurate approximation of the third component of the true error, which is in agreement with Theorem 4.4. However, the transient error estimate $\mathbf{E}^{\mathcal{R}}+\mathbf{E}^{\mathcal{N}}$ accurately approximates all components of the true error with global effectivity indices staying close to unity at all times and converging to unity under mesh refinement which is in full agreement with the theory.

TABle 5.9. Componentwise $L^{2}(\Omega)$-errors $\|\mathbf{e}\|^{*},\left\|\mathbf{e}-\mathbf{E}^{\mathcal{R}}\right\|^{*}$ and their order of convergence. Stationary global effectivity indices for Example 5.4 at $t=10^{-2}$.

\begin{tabular}{|c|c|c|c|c|c|c|}
\hline$p$ & $N$ & $\mid \mathbf{e} \|^{*}$ & order & $\left\|\mathbf{e}-\mathbf{E}^{\mathcal{R}}\right\|^{*}$ & order & $\theta^{*}$ \\
\hline \multirow{12}{*}{1} & \multirow{5}{*}{$10^{3}$} & $3.6332 e-3$ & \multirow{4}{*}{-} & {$[1.8285 e-4]$} & \multirow{4}{*}{ - } & 1.0210 \\
\hline & & $8.0813 e-1$ & & $4.5296 e-1$ & & 0.8467 \\
\hline & & $8.0813 e-1$ & & $4.5296 e-1$ & & 0.8467 \\
\hline & & $8.0813 e-1$ & & $4.5296 e-1$ & & 0.8467 \\
\hline & & {$[1.6201 e-3]$} & {$[1.9918]$} & {$[5.4438 e-5]$} & {$[2.9882]$} & 1.0141 \\
\hline & \multirow{4}{*}{$15^{3}$} & $3.6199 e-1$ & 1.9807 & $2.0361 e-1$ & 1.9720 & 0.8389 \\
\hline & & $3.6199 e-1$ & 1.9807 & $2.0361 e-1$ & 1.9720 & 0.8389 \\
\hline & & $3.6199 e-1$ & 1.9807 & $2.0361 e-1$ & 1.9720 & 0.8389 \\
\hline & & {$[9.1291 e-4]$} & 1.9940 & $\overline{[} 2.3039 e-5]$ & 2.9890 & $=1.0106$ \\
\hline & \multirow{3}{*}{$20^{3}$} & $2.0450 e-1$ & 1.9850 & $1.1536 e-1$ & 1.9750 & 0.8347 \\
\hline & & $2.0450 e-1$ & 1.9850 & $1.1536 e-1$ & 1.9750 & 0.8347 \\
\hline & & $2.0450 e-1$ & $1.9850]$ & {$[1.1536 e-1]$} & 1.9750 & 0.8347 \\
\hline \multirow{12}{*}{2} & \multirow{5}{*}{$10^{3}$} & $1.7317 e-5]$ & \multirow{4}{*}{-} & $1.2066 e-6$ & & 1.0140 \\
\hline & & $3.7744 e-3$ & & $2.2111 e-3$ & - & 0.8242 \\
\hline & & $3.7744 e-3$ & & $2.2111 e-3$ & - & 0.8242 \\
\hline & & $=3.7744 e-3$ & & $=2.2111 e-3$ & & $=0.8242$ \\
\hline & & $5.1369 e-6]$ & {$[2.9971]$} & {$[2.3878 e-7]$} & {$[3.9954]$} & 1.0098 \\
\hline & \multirow{4}{*}{$15^{3}$} & $1.1230 e-3$ & 2.9898 & $6.5783 e-4$ & 2.9899 & 0.8194 \\
\hline & & $1.1230 e-3$ & 2.9898 & $6.5783 e-4$ & 2.9899 & 0.8194 \\
\hline & & $=1.1230 e-3$ & 2.9898 & {$[6.5783 e-4$} & 2.9899 & $=0.8194$ \\
\hline & & $=2.1688 e-6$ & 2.9974 & {$[7.5630 e-8]$} & $3.9964]$ & $=1.0075$ \\
\hline & \multirow{3}{*}{$20^{3}$} & $4.7497 e-4$ & 2.9911 & $2.7856 e-4$ & 2.9871 & 0.8166 \\
\hline & & $4.7497 e-4$ & 2.9911 & $2.7856 e-4$ & 2.9871 & 0.8166 \\
\hline & & $4.7497 e-4$ & $2.9911]$ & {$[2.7856 e-4$} & 2.9871 & 0.8166 \\
\hline \multirow{12}{*}{3} & \multirow{5}{*}{$10^{3}$} & $6.2744 e-8$ & \multirow{4}{*}{ - } & $8.9829 e-9$ & \multirow{4}{*}{-} & 1.0041 \\
\hline & & $1.3640 e-5$ & & $8.3564 e-6$ & & 0.8021 \\
\hline & & $1.3640 e-5$ & & $8.3564 e-6$ & & 0.8021 \\
\hline & & $=1.3640 e-5$ & & $8.3564 e-6$ & & $=0.8021]$ \\
\hline & & $=1.2349 e-8$ & $4.0089]$ & {$[1.1839 e-9]$} & {$[4.9980]$} & $=1.0049$ \\
\hline & \multirow{4}{*}{$15^{3}$} & $2.6791 e-6$ & 4.0139 & $1.6165 e-6$ & 4.0515 & 0.8051 \\
\hline & & $2.6791 e-6$ & 4.0139 & $1.6165 e-6$ & 4.0515 & 0.8051 \\
\hline & & $=2.6791 e-6$ & 4.0139 & {$[1.6165 e-6]$} & 4.0515 & $=0.8051$ \\
\hline & & $=3.9033 e-9$ & 4.0036 & $2.8103 e-10$ & {$[4.9989]$} & $=1.0045$ \\
\hline & \multirow{3}{*}{$20^{3}$} & $8.4679 e-7$ & 4.0037 & $5.0859 e-7$ & 4.0197 & 0.8052 \\
\hline & & $8.4679 e-7$ & 4.0037 & $5.0859 e-7$ & 4.0197 & 0.8052 \\
\hline & & $8.4679 e-7$ & 4.0037 & $5.0859 e-7$ & 4.0197 & 0.8052 \\
\hline
\end{tabular}


TABLe 5.10. $L^{2}$-errors $\|\mathbf{e}\|_{2, \Omega},\left\|\mathbf{e}-\mathbf{E}^{\mathcal{R}}\right\|_{2, \Omega}$ and their order of convergence. Transient global effectivity indices for Example 5.4 at $t=10^{-2}$.

\begin{tabular}{|c|c|cc|cc|c|}
\hline$p$ & $N$ & $\|\mathbf{e}\|$ & order & $\left\|\mathbf{e}-\mathbf{E}^{\mathcal{R}}\right\|$ & order & $\theta$ \\
\hline \hline & $10^{3}$ & $1.3997 e-0$ & - & $7.8455 e-1$ & - & 0.8467 \\
1 & $15^{3}$ & $6.2698 e-1$ & 1.981 & $3.5267 e-1$ & 1.972 & 0.8389 \\
& $20^{3}$ & $3.5420 e-1$ & 1.985 & $1.9981 e-1$ & 1.975 & 0.8347 \\
\hline \hline & $10^{3}$ & $6.5374 e-3$ & - & $3.8298 e-3$ & - & 0.8242 \\
2 & $15^{3}$ & $1.9451 e-3$ & 2.99 & $1.1394 e-3$ & 2.99 & 0.8194 \\
& $20^{3}$ & $8.2268 e-4$ & 2.991 & $4.8247 e-4$ & 2.987 & 0.8166 \\
\hline \hline \multirow{3}{*}{3} & $10^{3}$ & $2.3624 e-5$ & - & $1.4474 e-5$ & - & 0.8021 \\
& $15^{3}$ & $4.6404 e-6$ & 4.014 & $2.7999 e-6$ & 4.052 & 0.8051 \\
& $20^{3}$ & $1.4667 e-6$ & 4.004 & $8.8090 e-7$ & 4.02 & 0.8052 \\
\hline
\end{tabular}

TABle 5.11. Componentwise $L^{2}(\Omega)$-errors $\|\mathbf{e}\|^{*},\left\|\mathbf{e}-\mathbf{E}^{\mathcal{R}}-\mathbf{E}^{\mathcal{N}}\right\|^{*}$ and their order of convergence. Transient global effectivity indices for Example 5.4 at $t=10^{-2}$.

\begin{tabular}{|c|c|c|c|c|c|c|}
\hline$p$ & $N$ & $\mid \mathbf{e} \|^{*}$ & order & $\left\|\mathbf{e}-\mathbf{E}^{\mathcal{R}}-\mathbf{E}^{\mathcal{N}}\right\|^{*}$ & order & $\theta^{*}$ \\
\hline \multirow{12}{*}{1} & \multirow{5}{*}{$10^{3}$} & {$[3.6332 e-3]$} & \multirow{4}{*}{-} & {$[1.8285 e-4]$} & \multirow{4}{*}{-} & {$[1.0210]$} \\
\hline & & $8.0813 e-1$ & & $6.6799 e-2$ & & 0.9926 \\
\hline & & $8.0813 e-1$ & & $6.6799 e-2$ & & 0.9926 \\
\hline & & $8.0813 e-1$ & & $6.6799 e-2$ & & 0.9926 \\
\hline & & {$[1.6201 e-3]$} & {$[1.9918]$} & {$[5.4438 e-5]$} & {$[2.9882]$} & {$[1.0141]$} \\
\hline & \multirow{4}{*}{$15^{3}$} & $3.6199 e-1$ & 1.9807 & $2.5398 e-2$ & 2.3850 & 0.9902 \\
\hline & & $3.6199 e-1$ & 1.9807 & $2.5398 e-2$ & 2.3850 & 0.9902 \\
\hline & & {$[3.6199 e-1$} & 1.9807 & $2.5398 e-2$ & [2.3850 & {$[0.9902]$} \\
\hline & & {$[9.1291 e-4]$} & {$[1.9940]$} & {$[2.3039 e-5]$} & {$[2.9890]$} & {$[1.0106]$} \\
\hline & \multirow{3}{*}{$20^{3}$} & $2.0450 e-1$ & 1.9850 & $1.3095 e-2$ & 2.3025 & 0.9894 \\
\hline & & $2.0450 e-1$ & 1.9850 & $1.3095 e-2$ & 2.3025 & 0.9894 \\
\hline & & $2.0450 e-1$ & 1.9850 & $1.3095 e-2$ & [2.3025] & 0.9894 \\
\hline \multirow{12}{*}{2} & \multirow{5}{*}{$10^{3}$} & {$[1.7317 e-5]$} & \multirow{4}{*}{ - } & $1.2066 e-6$ & & {$[1.0140]$} \\
\hline & & $3.7744 e-3$ & & $4.3774 e-4$ & - & 0.9867 \\
\hline & & $3.7744 e-3$ & & $4.3774 e-4$ & - & 0.9867 \\
\hline & & {$[3.7744 e-3]$} & & $4.3774 e-4$ & & {$[0.9867]$} \\
\hline & & {$[5.1369 e-6]$} & {$[2.9971]$} & {$[2.3878 e-7$} & {$[3.9954]$} & {$[1.0098]$} \\
\hline & \multirow{4}{*}{$15^{3}$} & $1.1230 e-3$ & 2.9898 & $1.0313 e-4$ & 3.5653 & 0.9880 \\
\hline & & $1.1230 e-3$ & 2.9898 & $1.0313 e-4$ & 3.5653 & 0.9880 \\
\hline & & {$[1.1230 e-3]$} & 2.9898 & $1.0313 e-4$ & [3.5653] & {$[0.9880]$} \\
\hline & & {$[2.1688 e-6]$} & $=2.9974]$ & {$[7.5630 e-8]$} & {$[3.9964]$} & {$[1.0075]$} \\
\hline & \multirow{3}{*}{$20^{3}$} & $4.7497 e-4$ & 2.9911 & $3.8389 e-5$ & 3.4352 & 0.9889 \\
\hline & & $4.7497 e-4$ & 2.9911 & $3.8389 e-5$ & 3.4352 & 0.9889 \\
\hline & & {$[4.7497 e-4]$} & [2.9911] & $3.8389 e-5$ & [3.4352] & [0.9889 \\
\hline \multirow{12}{*}{3} & \multirow{5}{*}{$10^{3}$} & {$[6.2744 e-8]$} & \multirow{4}{*}{-} & {$[8.9829 e-9$} & \multirow{4}{*}{-} & {$[1.0041]$} \\
\hline & & $1.3640 e-5$ & & $2.8570 e-6$ & & 0.9702 \\
\hline & & $1.3640 e-5$ & & $2.8570 e-6$ & & 0.9702 \\
\hline & & {$[1.3640 e-5]$} & & {$[2.8570 e-6$} & & {$[0.9702]$} \\
\hline & & {$[1.2349 e-8]$} & {$[4.0089]$} & {$[1.1839 e-9]$} & {$[4.9980]$} & {$[1.0049]$} \\
\hline & \multirow{3}{*}{$15^{3}$} & $2.6791 e-6$ & 4.0139 & $3.9691 e-7$ & 4.8681 & 0.9809 \\
\hline & & $2.6791 e-6$ & 4.0139 & $3.9691 e-7$ & 4.8681 & 0.9809 \\
\hline & & {$[2.6791 e-6]$} & 4.0139 & {$[3.9691 e-7]$} & [4.8681] & {$[0.9809$} \\
\hline & \multirow{4}{*}{$20^{3}$} & {$[3.9033 e-9]$} & {$[4.0036]$} & {$[2.8103 e-10]$} & {$[4.9989]$} & {$[1.0045]$} \\
\hline & & $8.4679 e-7$ & 4.0037 & $1.0018 e-7$ & 4.7855 & 0.9851 \\
\hline & & $8.4679 e-7$ & 4.0037 & $1.0018 e-7$ & 4.7855 & 0.9851 \\
\hline & & {$[8.4679 e-7]$} & 4.0037 & $1.0018 e-7$ & [4.7855] & {$[0.9851]$} \\
\hline
\end{tabular}


TABLe 5.12. $L^{2}$-errors $\|\mathbf{e}\|_{2, \Omega},\left\|\mathbf{e}-\mathbf{E}^{\mathcal{R}}-\mathbf{E}^{\mathcal{N}}\right\|_{2, \Omega}$ and their order of convergence. Transient global effectivity indices for Example 5.4 at $t=10^{-2}$.

\begin{tabular}{|c|c|cc|cc|c|}
\hline$p$ & $N$ & $\|\mathbf{e}\|$ & order & $\left\|\mathbf{e}-\mathbf{E}^{\mathcal{R}}-\mathbf{E}^{\mathcal{N}}\right\|$ & order & $\theta$ \\
\hline \hline & $10^{3}$ & $1.3997 e-0$ & - & $1.1570 e-1$ & - & 0.9926 \\
1 & $15^{3}$ & $6.2698 e-1$ & 1.981 & $4.3990 e-2$ & 2.385 & 0.9902 \\
& $20^{3}$ & $3.5420 e-1$ & 1.985 & $2.2682 e-2$ & 2.303 & 0.9894 \\
\hline \hline & $10^{3}$ & $6.5374 e-3$ & - & $7.5819 e-4$ & - & 0.9867 \\
2 & $15^{3}$ & $1.9451 e-3$ & 2.99 & $1.7863 e-4$ & 3.565 & 0.988 \\
& $20^{3}$ & $8.2268 e-4$ & 2.991 & $6.6492 e-5$ & 3.435 & 0.9889 \\
\hline \hline & $10^{3}$ & $2.3624 e-5$ & - & $4.9485 e-6$ & - & 0.9702 \\
3 & $15^{3}$ & $4.6404 e-6$ & 4.014 & $6.8746 e-7$ & 4.868 & 0.9809 \\
& $20^{3}$ & $1.4667 e-6$ & 4.004 & $1.7352 e-7$ & 4.785 & 0.9851 \\
\hline
\end{tabular}

\section{Conclusions}

In this paper, we investigated the DG method for linear symmetrizable hyperbolic systems with the enriched polynomial space $\mathcal{P}_{p}, \mathbb{P}_{p} \subset \mathcal{P}_{p} \subset \mathbb{P}_{p+1}$, and modified $L_{2}$-projections to approximate the initial and boundary conditions. For analysis, we symmetrized both the given system and its discretization and showed that the results of 8 can be applied to the resulting symmetric system. Thus, we showed that, for a local problem, the leading term of the discretization error lies in a polynomial subspace spanned by a linear combination of Legendre polynomials of order $p$ and $p+1$. We also established that a projection of the DG error into $\bigcap_{i=1}^{d} \mathcal{R}\left(\left(\mathbf{A}_{i}^{s_{i}}\right)^{t}\right)$, $s_{i}=+,-$, is $\mathcal{O}\left(h^{p+2}\right)$ superconvergent at Radau points.

We further constructed efficient and asymptotically exact implicit residual-based a posteriori error estimates where we split the leading term of the discretization error into two parts and estimated each part separately by solving a relatively small system of equations based on the local residual of the PDE. For systems with invertible coefficient matrices, the error estimates are obtained by solving a stationary problem, while, for general systems, part of the error is computed by solving a local transient system of equations. In contrast to the symmetric case, the splitting is not orthogonal, and the test function spaces for the error estimation are now based on the range, respectively, null space, of the transpose of each flux matrix, thus we have to solve local Petrov-Galerkin problems to compute an error estimate. We applied the results of $[8]$ on the asymptotic behavior of the DG error for symmetric systems to establish asymptotic correctness of the error estimates for symmetrized systems having smooth solutions. Finally, we presented computational results for several linear systems such as Maxwell's equations and the acoustic problem in two and three space dimensions.

We note that, at this point in time, we are not able to prove the asymptotic exactness of our global a posteriori error estimates for hyperbolic systems. However, computational results suggest that the global a posteriori error estimates are asymptotically exact for smooth solutions. Guided by the error analysis for the one-dimensional kinematic wave equation [3, we plan to prove the convergence of 
the global error estimate in the near future. Several other challenges such as nonlinear hyperbolic systems, other numerical fluxes and unstructured meshes remain to be addressed. Currently, we are investigating the behavior of DG errors when Lax-Friedrichs flux [10] is applied.

\section{REFERENCES}

[1] M. Abramowitz and I. A. Stegun, Handbook of mathematical functions, Dover, New York, 1965.

[2] S. Adjerid and M. Baccouch, The discontinuous Galerkin method for two-dimensional hyperbolic problems Part I: Superconvergence error analysis, Journal of Scientific Computing 33 (2007), 75-113. MR 2338333 (2008j:65189)

[3] _ Asymptotically exact a posteriori error estimates for a one-dimensional linear hyperbolic problem, submitted, 2009.

[4] _ The discontinuous Galerkin method for two-dimensional hyperbolic problems Part II: A Posteriori error estimation, Journal of Scientifc Computing 38 (2009), 15-49. MR 2472217

[5] S. Adjerid, K. D. Devine, J. E. Flaherty, and L. Krivodonova, A posteriori error estimation for discontinuous Galerkin solutions of hyperbolic problems, Computer Methods in Applied Mechanics and Engineering 191 (2002), 1097-1112. MR 1877682(2002k:65137)

[6] S. Adjerid and T. C. Massey, A posteriori discontinuous finite element error estimation for two-dimensional hyperbolic problems, Computer Methods in Applied Mechanics and Engineering 191 (2002), 5877-5897. MR1942623 (2003j:65110)

[7] _ Superconvergence of discontinuous finite element solutions for nonlinear hyperbolic problems, Computer Methods in Applied Mechanics and Engineering 195 (2006), 3331-3346. MR2220922 (2006k:65304)

[8] S. Adjerid and T. Weinhart, Asymptotically exact discontinuous Galerkin error estimates for linear symmetric hyperbolic systems, submitted, 2009.

[9] _ Discontinuous Galerkin error estimation for linear symmetric hyperbolic systems, Computer Methods in Applied Mechanics and Engineering 198 (2009), 3113-3129. MR2567860

[10] , Discontinuous Galerkin error estimation for linear symmetric hyperbolic systems with Lax Friedricks flux, in preparation, 2009.

[11] S. Benzoni-Gavage and D. Serre, Multidimensional hyperbolic partial differential equations, Oxford University Press, 2007. MR 2284507 (2008k:35002)

[12] Y. Cheng and C.-W. Shu, Superconvergence and time evolution of discontinuous Galerkin finite element solutions, Journal of Computational Physics 227 (2008), 9612-9627. MR2467636 (2009k:65182)

[13] _ Superconvergence of local discontinuous Galerkin methods for one-dimensional convection-diffusion equations, Computers \& Structures 87 (2009), 630-641.

[14] _ Superconvergence of discontinuous Galerkin and local discontinuous galerkin schemes for linear hyperbolic and convection-diffusion equations in one space dimension, SIAM Journal on Numerical Analysis 47 (2010), 4044-4072. MR2585178

[15] B. Cockburn, A simple introduction to error estimation for nonlinear hyperbolic conservation laws, Proceedings of the 1998 EPSRC Summer School in Numerical Analysis, SSCM, volume 26 of the Graduate Student's Guide for Numerical Analysis, pages 1-46 (Berlin), Springer, 1999. MR 1715030

[16] B. Cockburn and P. A. Gremaud, Error estimates for finite element methods for nonlinear conservation laws, SIAM Journal on Numerical Analysis 33 (1996), 522-554. MR1388487 (97e:65096)

[17] B. Cockburn, S. Hou, and C. W. Shu, TVB Runge-Kutta local projection discontinuous Galerkin finite element method for conservation laws IV: The multidimensional case, Math. Comp. 54 (1990), 545-581. MR1010597 (90k:65162)

[18] B. Cockburn, S. Y. Lin, and C. W. Shu, TVB Runge-Kutta local projection discontinuous Galerkin methods of scalar conservation laws III: One dimensional systems, Journal of Computational Physics 84 (1989), 90-113. MR1015355 (90k:65161)

[19] B. Cockburn and C. W. Shu, The Runge-Kutta local projection p1-discontinuous Galerkin method for scalar conservation laws, RAIRO Modél. Math. Anal. Numér. 25 (1991), 337-361. MR:1103092(92e:65128) 
[20] J. R. Dormand and P. J. Prince, A family of embedded Runge-Kutta formula, Journal of Computational and Applied Mathematics 6 (1980), no. 1, 19-26. MR.568599 (81g:65098)

[21] R. Hartmann and P. Houston, Adaptive discontinuous Galerkin finite element methods for nonlinear hyperbolic conservations laws, SIAM J. Sci. Comput. 24 (2002), 979-1004. MR:1950521(2004b:65149)

[22] L. Krivodonova and J. E. Flaherty., Error estimation for discontinuous Galerkin solutions of two-dimensional hyperbolic problems, Advances in Computational Mathematics 19 (2003), 57-71. MR 1973459 (2004e:65112)

[23] M. Larson and T. Barth, A posteriori error estimation for adaptive discontinuous Galerkin approximation of hyperbolic systems, Proc. International Symposium on Discontinuous Galerkin Methods Theory, Computation and Applications (Berlin) (B. Cockburn, G. E. Karniadakis, and C. W. Shu, eds.), Springer, 2000. MR 1842194 (2002f:65163)

[24] P. LeSaint and P. Raviart, On a finite element method for solving the neutron transport equations, Mathematical Aspects of Finite Elements in Partial Differential Equations (New York) (C. de Boor, ed.), Academic Press, 1974, pp. 89-145. MR0658142 (58:31918)

[25] W. H. Reed and T. R. Hill, Triangular mesh methods for the neutron transport equation, Tech. Report LA-UR-73-479, Los Alamos Scientific Laboratory, Los Alamos, 1973.

[26] Uriel G. Rothblum, A representation of the Drazin inverse and characterizations of the index, SIAM Journal on Applied Mathematics 31 (1976), no. 4, 646-648. MR0422303 (54:10294)

[27] J. C. Tannehill, D. A. Anderson, and R. H. Pletcher, Computational fluid mechanics and heat transfer, 2nd ed., Taylor \& Francis, 1997.

Department of Mathematics, Virginia Polytechnic Institute and State University, Blacksburg, Virginia 24061

Department of Applied Mathematics, University of Twente, 7500 AE Enschede, The NETHERLANDS 